\title{
A Quantitative Model of The Cerebral Windkessel as A Frequency-Sensitive Filter to Provide A Novel Understanding of Intracranial Dynamics
}

Michael Egnor ( $\square$ michael.egnor@stonybrookmedicine.edu )

Stony Brook University Hospital

\section{Liu Yang}

Stony Brook University

Racheed Mani

Stony Brook University Hospital

\section{Susan Fiore}

Stony Brook University Hospital

Petar Djuric

Stony Brook University

\section{Research Article}

Keywords: mathematical modeling, cerebral windkessel, cerebral blood flow, cerebrospinal fluid, brain edema, hydrocephalus, intracranial dynamics

Posted Date: February 7th, 2022

DOI: https://doi.org/10.21203/rs.3.rs-1310634/v1

License: (c) (1) This work is licensed under a Creative Commons Attribution 4.0 International License. Read Full License 


\section{A quantitative model of the cerebral windkessel as a frequency-sensitive filter to provide a novel understanding of intracranial dynamics}

Michael Egnor ${ }^{1}$, Liu Yang ${ }^{2}$, Racheed Mani ${ }^{1}$, Susan Fiore ${ }^{1}$, Petar Djuric ${ }^{2}$

${ }^{1}$ Department of Neurological Surgery, Stony Brook University Hospital, Stony Brook, New York, United States of America

${ }^{2}$ Department of Electrical and Computer Engineering, Stony Brook University, Stony Brook, New York, United States of America

Corresponding Author:

michael.egnor@stonybrookmedicine.edu

Keywords: mathematical modeling; cerebral windkessel; cerebral blood flow; cerebrospinal fluid; brain edema; hydrocephalus; intracranial dynamics 


\section{Abstract}

Background: Traditional models of intracranial dynamics fail to capture several important features of the cerebral windkessel. Experiments show that the cerebral windkessel is a bandstop filter tuned to the heart rate, which is not consistent with existing pressure-volume compartment models. Flow MRI studies in humans reveal expansion and relaxation of the cranial contents during the cardiac cycle that continuously opposes arterial expansion and relaxation, and we suggest this is the way the windkessel is implemented in the cranium. This systolic-diastolic brain motion is analogous to the loading and unloading of the capacitor plates in the model circuit.

Methods: In this study, we use exogenous input autoregressive (ARX) modeling of a simple band-stop electrical circuit to model the cerebral windkessel of normal dogs. We compare the circuit waveforms to waveforms measured in dogs, and we examine the dynamics that underlie the cerebral windkessel.

Results: Our ARX analysis shows close agreement between the circuit and the windkessel, which implies similar physical mechanisms of pulsation suppression. This correspondence between physiological data and circuit dynamics suggests that the cerebral windkessel is a frequency-sensitive band-stop filter for the passage of arterial power through the cranium. Another perspective from which to understand the windkessel is to consider the smooth offset and the pulsatile components of cerebral arterial flow as DC and AC power respectively. In the cranium, DC power of cerebral blood flow passes through the capillary bed to the veins and AC power is diverted through the CSF to the veins. This windkessel mechanism optimizes smooth capillary flow and minimizes potentially damaging capillary pulsatility. 
Conclusions: Our windkessel model provides substantial new insights into intracranial dynamics, cerebral blood flow and capillary circulation, and it provides a new framework for the study and treatment of several common disorders of intracranial dynamics such as cerebral edema, hydrocephalus, pseudotumor cerebri, and Cushing's reflex.

\section{Background}

Although arterial blood flow is pulsatile, capillary flow is nearly pulseless [1]. Elastic expansion of arteries stores a portion of the systolic bolus and releases it into the distal circulation in diastole. Arterial storage and release of a portion of the systolic bolus clips systolic flow and boosts diastolic flow and converts pulsatile arterial flow into pulseless flow in the microvasculature. This suppression of capillary pulsatility is the windkessel [2].

The physiological rationale of smooth capillary flow seems clear. Significant capillary pulsatility raises capillary hydrostatic pressure and risks edema, and radial pulsatile stress may rupture capillary endothelium, which is one cell thick. The general properties of the systemic windkessel were known to physiologists in the 18th and 19th century [3], and Frank [4], in 1899 proposed a quantitative model of the aortic windkessel, using measurements of arterial resistance and compliance. Frank's model failed to accurately predict the relationship between arterial pressure and flow, and in 1971, Westerhof [5] published more accurate simulations using the characteristic impedance of the aorta, which includes arterial inertance, as well as resistance and compliance, and takes into account the passage of the arterial expansion and relaxation along the length of the artery. 
In the cranium, however, the rigid skull and incompressible fluids constrain expansion and relaxation of arteries, which precludes a cerebral windkessel mechanism identical to the aortic windkessel.

During the past two decades, investigators have characterized the cerebral windkessel using the transfer function between the arterial blood pressure (ABP) pulse and the intracranial pressure (ICP) pulse [6], with attention to the cerebral input impedance rather than to the aortic characteristic impedance used by Westerhof. The cerebral windkessel transfer function has been analyzed in normal dynamics and in intracranial hypertension [6] as well as in hydrocephalus in dogs [7] and in humans [8] .

The goal of this study is to investigate the dynamics underlying the cerebral windkessel.

\section{Characteristics of the cerebral windkessel}

Transfer function analysis of the arterial blood pressure (ABP) pulse and the ICP pulse shows that the cranium is a band-stop (notch) filter centered at the heart rate [6] [Fig 1]. This frequency domain characteristic of the ICP pulse correlates with the time domain observation that the ICP pulse is normally of minimal amplitude [1].

Fig 1. Graphs showing the transfer function of the windkessel in an experimental dog (from Wagshul et. al. [6] with permission)

(A) The amplitude transfer function, which shows the high impedance stop band at the heart rate. This represents the suppression of the arterial pulse in the capillaries of the 
brain parenchyma. (B) The phase transfer function, which shows a phase transition near the cardiac fundamental, which is characteristic of a pulsation absorber tuned to high impedance resonance at the heart rate. The open circles mark the fundamental (heart rate) harmonic, and the second and third harmonics. The electrical analogue of the cerebral windkessel is a tank circuit with capacitance and inductance in parallel (see text).

There is a phase transition near the central frequency of the notch [6], which implies that the windkessel band-stop filter is dependent on high impedance resonant dynamics in the cranium. The notch corresponds to a phase transition in the phase transfer function.

The ABP-ICP phase transfer function of the fundamental heart rate frequency in normal dogs is positive by about 60 degrees, which means that in the time domain the ICP pulse normally precedes the carotid ABP pulse [Fig $2 A]$. This phase lead of ICP to ABP is not the result of an ICP phase lag of nearly a full cycle from the previous ABP pulse. This can be seen from the recording of asystoles in the same experimental animals in which the phase lead was measured, which show no ICP pulse in the period of skipped arterial beats [Fig 2B]. The ICP pulse normally occurs slightly prior to the ABP pulse, which is profoundly counterintuitive and inconsistent with pressure-volume models of the cerebral windkessel. The phase lead of output (ICP) with respect to input (ABP) is consistent with a damped band stop filter in steady state oscillation [2].

Fig 2A) The experimentally measured ICP lead with respect to the arterial pulse in a dog from Wagshul et al.[6]

Fig 2B) Graphical evidence that the phase lead of the ICP with respect to the ABP is real, and not merely a five-sixth cycle lag from the previous ABP pulse. 
Experimental raising of intracranial pressure and experimental and clinical hydrocephalus obliterate the band-stop notch on the transfer function, and restoration of normal dynamics restores the notch [6-9].

In the time domain, the amplitude of the intracranial pressure (ICP) pulse (the ICP difference from diastole to systole) is normally at a minimum. Intracranial compliance increases and decreases with lowering and raising ICP, respectively, yet both experimental lowering and raising ICP increase the ICP pulse amplitude [10,11], which is inconsistent with traditional pressure-volume models.

There is normally minimal reflectance of the arterial pulse retrograde through the arterial tree [12]. Given that capillary perfusion is smooth, minimal retrograde arterial reflectance means that the arterial pulse is transmitted through the cranium via a pathway other than the cerebral microcirculation.

\section{Pressure-volume models are inadequate to describe the cerebral windkessel}

Conventional models of the intracranial dynamics, such as those of Ursino and others [13-15], are pressure-volume compartment models of the cranium, and use the precepts of the MonroKellie doctrine to simulate intracranial pressure-volume dynamics. While these models successfully describe several aspects of intracranial dynamics, such as intracranial pressure waves and the paradoxical elevation of ICP with systemic hypotension, these pressure-volume models address the cerebral windkessel itself only tangentially - the focus of these models is on simulation of bulk flow dynamics. 
These models simulate the windkessel by diverting the arterial pulse away from the capillaries via parallel capacitance without consideration of intracranial inertance as a component of load impedance. These models are low-pass filters and would not produce the resonant behavior empirically observed in the cerebral windkessel. Furthermore, compartment models cannot reproduce the normal phase lead of ICP with respect to ABP as noted above.

The inadequacy of pressure-volume compartment models to account for these empirical characteristics of the cerebral windkessel suggests a new model of the cerebral windkessel and a novel understanding of intracranial dynamics.

\section{Goals of this study}

In accordance with these considerations, we have modeled the cerebral windkessel using an electrical anti-resonant band-stop filter implemented by an electrical circuit tuned to the heart rate frequency with series resistance and parallel inductance and capacitance. The goals of our study are:

1) To determine the configuration of resistive and reactive circuit elements necessary to produce the experimentally observed anti-resonant band-stop filter of the cerebral windkessel.

2) To correlate the circuit dynamics quantitatively with experimental data from dogs.

3) To use the simplified dynamics of the electrical circuit model to gain insight into the complex anatomical and physiological mechanism by which the windkessel is implemented in the cranium.

The simplest implementation of an electrical band-stop filter is a tank circuit with a parallel capacitor and inductor, and this is the basis for our model. 


\section{Methods}

\section{The tank circuit model of the cerebral windkessel}

The ABP pulse and the ICP pulse are each analogous to a simple harmonic oscillator driven at the heart rate [2]. The pulse is the cyclic exchange of kinetic and potential energy, in a manner similar to a mass oscillating on a spring or electrons oscillating in a circuit. The electrical analogue to the pulse is $A C$ flow in a circuit with an inductor (which stores and releases kinetic energy), a capacitor (which stores and releases potential energy), and a resistor (which dissipates energy). Smooth blood flow is the DC offset, corresponding to mean cerebral blood flow (CBF) driven by the mean arterial pressure (MAP). Pulsations of blood, CSF and brain parenchyma correspond to AC flow. Power is the rate of energy flow, and DC and AC power in our model together correspond to the rate of smooth and pulsatile energy flow in total CBF. The CBF in large arteries and veins entering and leaving the cranium is a composite of DC and AC power, although the cerebral capillaries are ordinarily the path for only DC power. CSF formation is a miniscule component of DC power that flows in the CSF path, and CSF formation plays no role in this model, except as the means by which CSF volume and thus coupling between arterial and venous pulsations is maintained. In the fluids in the cranium, pressure is evenly distributed (Bernoulli's principle) but flow can travel in different paths with different impedances. This corresponds to a parallel (tank) electrical circuit.

In the cranium, the transfer function analysis of the ABP and ICP shows that the windkessel is a band stop filter [6]. A band stop filter is a system that selectively suppresses signals with frequencies within a given bandwidth. In mechanics, a band stop filter is a dynamic vibration 
absorber [2]. In an electrical system it is a tank circuit. In acoustics it is a cavity resonator. The dynamic principles underling band stop filters are the same: circulating motion in an absorber path continuously opposes oscillatory motion in the main path. A table of equivalence is given for the implementation of band stop filter dynamics in a variety of systems (see Table 1).

With these assumptions (summarized in Table 1), we model CBF and intracranial dynamics using an equivalent tank circuit (Fig 3). We compare the circuit waveforms to waveforms measured in dogs, and we examine the dynamics that underlie the cerebral windkessel.

\section{Table 1}

Equivalence of mechanical, electrical, and cerebral windkessel parameters.

\begin{tabular}{|c|c|c|c|c|c|}
\hline Parameter & $\begin{array}{l}\text { Mechanical } \\
\text { (dynamic } \\
\text { vibration } \\
\text { absorber) }\end{array}$ & $\begin{array}{l}\text { Electrical } \\
\text { (tank } \\
\text { circuit) }\end{array}$ & $\begin{array}{l}\text { Cerebral } \\
\text { windkessel }\end{array}$ & Windkessel symbol/equation & $\begin{array}{l}\text { Windkessel } \\
\text { Units (SI) }\end{array}$ \\
\hline Force & Force & Voltage & Pressure & $\begin{array}{l}P \text { is pressure which corresponds to } \\
\text { potential energy density of intracranial } \\
\text { contents }\end{array}$ & $\mathrm{kg} \mathrm{m}^{-1} \mathrm{~s}^{-1}$ \\
\hline Velocity & Velocity & Current & $\begin{array}{l}\text { Volumetric } \\
\text { flow }\end{array}$ & Rate of volumetric flow & $m^{3} s^{-1}$ \\
\hline Displacement & Displacement & Charge & Volume & $\begin{array}{l}\text { Volume of fluid/tissue displaced. Virtually } \\
\text { all intracranial volume displacement } \\
\text { during the cardiac cycle is dependent on } \\
\text { displacement of venous blood. }\end{array}$ & $m^{3}$ \\
\hline $\begin{array}{l}\text { Forcing } \\
\text { frequency }\end{array}$ & $\begin{array}{l}\text { Forcing } \\
\text { frequency }\end{array}$ & $\begin{array}{l}\mathrm{AC} \\
\text { frequency }\end{array}$ & Heart rate & $\begin{array}{l}\omega=2 \pi f: \text { the heart rate, expressed in } \\
\text { radians/sec }\end{array}$ & $s^{-1}$ (radians) \\
\hline $\begin{array}{l}\text { Natural (anti- } \\
\text { resonant) } \\
\text { frequency }\end{array}$ & $\begin{array}{l}\text { Natural (anti- } \\
\text { resonant) } \\
\text { frequency }\end{array}$ & $\begin{array}{l}\text { Natural } \\
\text { (anti- }\end{array}$ & $\begin{array}{l}\text { Natural (anti- } \\
\text { resonant) } \\
\text { frequency }\end{array}$ & $2 \pi f_{w k}=\omega_{w k}=\sqrt{\frac{1}{L C}-\frac{R_{C S F}^{2}}{L^{2}}}$ & $s^{-1}$ (radians) \\
\hline
\end{tabular}




\begin{tabular}{|c|c|c|c|c|c|}
\hline & & $\begin{array}{l}\text { resonant) } \\
\text { frequency }\end{array}$ & & $\begin{array}{l}\text { The frequency at which windkessel } \\
\text { impedance is pure resistance without } \\
\text { reactance and is at a local maximum. } \\
\text { When optimally tuned, the heart rate } \\
\text { equals the natural anti-resonant frequency } \\
\text { of the windkessel. This presupposes } \\
\text { physiological regulation of the windkessel. }\end{array}$ & \\
\hline Inertia & Mass & Inductance & Inertance & $\begin{array}{l}\qquad \frac{\Delta P}{\Delta \dot{V}} \\
\text { Ratio of pressure change to change in } \\
\text { volumetric flow. Corresponds to mass in a } \\
\text { mechanical oscillator. }\end{array}$ & $\begin{array}{l}\frac{\mathrm{kg} \mathrm{m}^{-1} \mathrm{~s}^{-1}}{\mathrm{~m}^{3} \mathrm{~s}^{-1}} \\
=k g \mathrm{~m}^{-4}\end{array}$ \\
\hline $\begin{array}{l}\text { Resistance in } \\
\text { main path } \\
\text { (opposition to } \\
\text { unaccelerated } \\
\text { motion) }\end{array}$ & $\begin{array}{l}\text { Resistance or } \\
\text { damping in } \\
\text { main } \\
\text { mass/spring }\end{array}$ & $\begin{array}{l}\text { Resistance } \\
\text { in main } \\
\text { (series) line }\end{array}$ & $\begin{array}{l}\text { Capillary } \\
\text { resistance }\end{array}$ & $\begin{array}{l}\text { Maip } \\
\text { Mainly viscous resistance to longitudinal } \\
\text { flow of blood in capillaries. }\end{array}$ & $\mathrm{kg} \mathrm{m}^{-4} \mathrm{~s}^{-1}$ \\
\hline $\begin{array}{l}\text { Resistance in } \\
\text { absorber path } \\
\text { (opposition to } \\
\text { unaccelerated } \\
\text { motion) }\end{array}$ & $\begin{array}{l}\text { Resistance or } \\
\text { damping }\end{array}$ & Resistance & $\begin{array}{l}\text { Resistance or } \\
\text { damping }\end{array}$ & $\begin{array}{l}\qquad \frac{R_{C S F}}{\omega^{2} R_{C S F}{ }^{2} C^{2}+\left(\omega^{2} L C-1\right)^{2}} \\
\text { Corresponds to viscous resistance of } \\
\text { radial motion of blood in large intracranial } \\
\text { vessels, to resistance to oscillatory CSF } \\
\text { flow and to structural damping in vessel } \\
\text { walls and brain parenchyma. }\end{array}$ & $\mathrm{kg} \mathrm{m}^{-4} \mathrm{~s}^{-1}$ \\
\hline $\begin{array}{l}\text { Compliance } \\
\text { (reciprocal of } \\
\text { ratio of force } \\
\text { to } \\
\text { displacement) }\end{array}$ & $\begin{array}{l}\text { Reciprocal of } \\
\text { spring } \\
\text { constant }\end{array}$ & Capacitance & Compliance & $\begin{array}{l}\qquad \frac{\Delta V}{\Delta P} \\
\text { Ratio of volume change to pressure } \\
\text { change. Intracranial compliance over the } \\
\text { interval of the cardiac cycle is due to } \\
\text { compliance of arterial and venous walls } \\
\text { and to displacement of venous blood. }\end{array}$ & $\mathrm{kg}^{-1} \mathrm{~m}^{4} \mathrm{~s}^{2}$ \\
\hline $\begin{array}{l}\text { Reactance } \\
\text { (net } \\
\text { opposition to }\end{array}$ & $\begin{array}{l}\text { Mechanical } \\
\text { reactance }\end{array}$ & $\begin{array}{l}\text { Electrical } \\
\text { Reactance }\end{array}$ & $\begin{array}{l}\text { Reactance in } \\
\text { a fluid medium } \\
\text { with }\end{array}$ & $\begin{array}{l}\quad X_{C S F}=j\left[\frac{\omega C\left(-\omega^{2} L^{2}+\frac{L}{C}-R_{C S F}^{2}\right)}{\omega^{2} R_{C S F}^{2} C^{2}+\left(\omega^{2} L C-1\right)^{2}}\right] \\
\text { Reactance is the imaginary component of } \\
\text { impedance. It represents net opposition to }\end{array}$ & $\mathrm{kg} \mathrm{m}^{-4} \mathrm{~s}^{-1}$ \\
\hline
\end{tabular}




\begin{tabular}{|c|c|c|c|c|c|}
\hline $\begin{array}{l}\text { accelerated } \\
\text { motion) }\end{array}$ & & & $\begin{array}{l}\text { distributed } \\
\text { parameters. }\end{array}$ & $\begin{array}{l}\text { accelerated flow due to inertance and } \\
\text { compliance in the cranium. }\end{array}$ & \\
\hline $\begin{array}{l}\text { Inertial } \\
\text { reactance } \\
\text { (opposition to } \\
\text { accelerated } \\
\text { motion due to } \\
\text { inertia) }\end{array}$ & $\begin{array}{l}\text { Inertial } \\
\text { reactance }\end{array}$ & $\begin{array}{l}\text { Inductive } \\
\text { reactance }\end{array}$ & $\begin{array}{l}\text { Inertial } \\
\text { reactance }\end{array}$ & $\begin{array}{l}j \omega L \\
\text { Positive imaginary component of } \\
\text { impedance; represents net opposition to } \\
\text { accelerated flow due to inertance in } \\
\text { cranium. }\end{array}$ & $\mathrm{kg} \mathrm{m}^{-4} \mathrm{~s}^{-1}$ \\
\hline $\begin{array}{l}\text { Capacitive } \\
\text { reactance } \\
\text { (opposition to } \\
\text { accelerated } \\
\text { motion due to } \\
\text { elastance) }\end{array}$ & $\begin{array}{l}\text { Elastic } \\
\text { reactance }\end{array}$ & $\begin{array}{l}\text { Capacitive } \\
\text { reactance }\end{array}$ & $\begin{array}{l}\text { Capacitive } \\
\text { reactance }\end{array}$ & $\begin{array}{l}\frac{1}{j \omega C} \\
\text { Negative imaginary component of } \\
\text { impedance; represents net opposition to } \\
\text { accelerated flow due to compliance in } \\
\text { cranium. }\end{array}$ & $\mathrm{kg} \mathrm{m}^{-4} \mathrm{~s}^{-1}$ \\
\hline $\begin{array}{l}\text { Impedance } \\
\text { (complex ratio } \\
\text { of force to } \\
\text { velocity) }\end{array}$ & $\begin{array}{l}\text { Mechanical } \\
\text { impedance }\end{array}$ & Impedance & $\begin{array}{l}\text { Windkessel } \\
\text { impedance } \\
\left(Z_{w k}\right)\end{array}$ & $\begin{array}{l}\frac{R_{C S F}}{\omega^{2} R_{C S F}^{2} C^{2}+\left(\omega^{2} L C-1\right)^{2}} \\
+j\left[\frac{\omega C\left(-\omega^{2} L^{2}+\frac{L}{C}-R_{C S F}^{2}\right)}{\omega^{2} R_{C S F}^{2} C^{2}+\left(\omega^{2} L C-1\right)^{2}}\right] \\
\text { Net opposition to flow in the windkessel, } \\
\text { expressed as a complex quantity. }\end{array}$ & $\mathrm{kg} \mathrm{m}^{-4} \mathrm{~s}^{-1}$ \\
\hline $\begin{array}{l}\text { Impedance } \\
\text { magnitude }\end{array}$ & $\begin{array}{l}\text { Impedance } \\
\text { magnitude }\end{array}$ & $\begin{array}{l}\text { Impedance } \\
\text { magnitude }\end{array}$ & $\begin{array}{l}\text { Impedance } \\
\text { magnitude }\end{array}$ & $|Z|=\sqrt{R_{C S F}^{2}+X_{C S F}^{2}}$ & $\mathrm{~kg} \mathrm{~m}^{-4} \mathrm{~s}^{-1}$ \\
\hline $\begin{array}{l}\text { Impedance } \\
\text { phase }\end{array}$ & $\begin{array}{l}\text { Impedance } \\
\text { phase }\end{array}$ & $\begin{array}{l}\text { Impedance } \\
\text { phase }\end{array}$ & $\begin{array}{l}\text { Impedance } \\
\text { phase }\end{array}$ & $\begin{array}{l}\quad \theta_{w k}=\arctan \left[\frac{X_{C S F}}{R_{C S F}}\right] \\
\quad=\arctan \left[\frac{\omega C\left(-\omega^{2} L^{2}+\frac{L}{C}-R_{C S F}^{2}\right)}{R_{C S F}}\right] \\
\text { Impedance phase is the phase of } \\
\text { pressure with respect to flow. In the } \\
\text { transfer function of ABP to ICP, } \\
\text { impedance phase is the phase of the ICP } \\
\text { with respect to the ABP. Positive phase } \\
\text { represents an ICP lead, and negative } \\
\text { phase represents an ICP lag. }\end{array}$ & radians \\
\hline
\end{tabular}




\begin{tabular}{|c|c|c|c|c|c|}
\hline $\begin{array}{l}\text { Anti-resonant } \\
\text { natural } \\
\text { frequency }\end{array}$ & $\begin{array}{l}\text { Anti-resonant } \\
\text { natural } \\
\text { frequency }\end{array}$ & $\begin{array}{l}\text { Anti- } \\
\text { resonant } \\
\text { natural } \\
\text { frequency }\end{array}$ & $\begin{array}{l}\text { Anti-resonant } \\
\text { natural } \\
\text { frequency }\end{array}$ & $\begin{array}{l}\qquad \omega_{w k}=\sqrt{\frac{1}{L C}-\frac{R_{C S F}^{2}}{L^{2}}} \\
\text { Frequency at which complex intracranial } \\
\text { impedance is maximal and due to pure } \\
\text { resistance. }\end{array}$ & $s^{-1}$ (radians) \\
\hline Effectiveness & & & $\begin{array}{l}\text { Windkessel } \\
\text { effectiveness }\end{array}$ & $\begin{array}{l}\qquad R_{e f f}=\frac{L}{R_{C S F} C} \\
\text { A measure of optimal windkessel } \\
\text { effectiveness (i.e. maximal resistance) } \\
\text { when windkessel is tuned to the anti- } \\
\text { resonant heart rate } \omega_{w k} \text { and impedance is } \\
\text { purely resistive. }\end{array}$ & Dimensionless \\
\hline $\begin{array}{l}\text { Unaccelerated } \\
\text { motion (DC } \\
\text { offset) }\end{array}$ & $\begin{array}{l}\text { Unaccelerated } \\
\text { motion }\end{array}$ & $\begin{array}{l}\text { Direct } \\
\text { current }\end{array}$ & $\begin{array}{l}\text { DC flow, which } \\
\text { corresponds to } \\
\text { mean CBF }\end{array}$ & $\begin{array}{l}\text { DC flow: smooth flow of blood } \\
\text { corresponding to mean CBF. }\end{array}$ & $m^{3} s^{-1}$ \\
\hline $\begin{array}{l}\text { Accelerated } \\
\text { motion (AC) }\end{array}$ & $\begin{array}{l}\text { Accelerated } \\
\text { motion }\end{array}$ & $\begin{array}{l}\text { Alternating } \\
\text { current }\end{array}$ & $\begin{array}{l}\text { AC pressure } \\
\text { or flow }\end{array}$ & AC: pulsations in blood, CSF and tissues. & $m^{3} s^{-1}$ \\
\hline $\begin{array}{l}\text { Energy } \\
\text { (product of } \\
\text { force and } \\
\text { displacement) }\end{array}$ & $\begin{array}{l}\text { Mechanical } \\
\text { energy }\end{array}$ & $\begin{array}{l}\text { Electrical } \\
\text { energy }\end{array}$ & Energy & $\begin{array}{l}\text { Energy: net kinetic, potential (pressure } \\
\text { energy), gravitational and heat energy in } \\
\text { cranium. }\end{array}$ & $\begin{array}{l}\mathrm{kg} \mathrm{m}^{2} \mathrm{~s}^{-2}= \\
\text { Joules }\end{array}$ \\
\hline Pressure & $\begin{array}{l}\text { Energy } \\
\text { density }\end{array}$ & $\begin{array}{l}\text { Energy } \\
\text { density }\end{array}$ & $\begin{array}{l}\text { Energy density } \\
\text { (intracranial } \\
\text { pressure) }\end{array}$ & $\begin{array}{l}\mathrm{P} \text { is a measure of potential energy density } \\
\text { in cranium. }\end{array}$ & $\begin{array}{l}\frac{\mathrm{kg} \mathrm{m^{2 } \mathrm { s } ^ { - 2 }}}{\mathrm{m}^{3}}= \\
\qquad \mathrm{kg} \mathrm{m}^{-1} \mathrm{~s}^{-2} \\
\text { Unit is pascals. } \\
133 \text { pascals = } \\
1 \text { torr }\end{array}$ \\
\hline $\begin{array}{l}\text { Power (rate of } \\
\text { energy flow) }\end{array}$ & $\begin{array}{l}\text { Mechanical } \\
\text { power }\end{array}$ & $\begin{array}{l}\text { Electrical } \\
\text { power }\end{array}$ & $\begin{array}{l}\text { Power (rate of } \\
\text { energy flow) }\end{array}$ & $\begin{array}{l}\text { Power: a measure of the rate of energy } \\
\text { flow in cranium. }\end{array}$ & $\begin{array}{r}k g \mathrm{~m}^{2} \mathrm{~s}^{-3} \\
\text { Unit = Watts }\end{array}$ \\
\hline Main path & $\begin{array}{l}\text { Main mass } \\
\text { and spring }\end{array}$ & $\begin{array}{l}\text { Main series } \\
\text { line }\end{array}$ & Capillaries & $\begin{array}{l}\text { In windkessel theory, refers specifically to } \\
\text { longitudinal dimension of capillary lumina. }\end{array}$ & \\
\hline
\end{tabular}




\begin{tabular}{|c|c|c|c|c|}
\hline Absorber path & $\begin{array}{l}\text { Absorber } \\
\text { mass and } \\
\text { spring }\end{array}$ & $\begin{array}{l}\text { Parallel tank } \\
\text { circuit }\end{array}$ & $\begin{array}{l}\text { Extracapillary } \\
\text { tissue and } \\
\text { fluid (arterial } \\
\text { walls, brain } \\
\text { parenchyma, } \\
\text { ISF, CSF, } \\
\text { veins) }\end{array}$ & $\begin{array}{l}\text { Refers to radial dimension of capillary } \\
\text { lumina and to all fluids and tissues in } \\
\text { cranium except longitudinal dimension of } \\
\text { capillary lumina. For brevity, may be } \\
\text { referred to as the CSF path. }\end{array}$ \\
\hline $\begin{array}{l}\text { Main-to- } \\
\text { absorber link }\end{array}$ & $\begin{array}{l}\text { Main-to- } \\
\text { absorber link }\end{array}$ & $\begin{array}{l}\text { Conductor } \\
\text { connecting } \\
\text { main series } \\
\text { line to tank } \\
\text { circuit }\end{array}$ & $\begin{array}{l}\text { Extracapillary } \\
\text { fluids }\end{array}$ & $\begin{array}{l}\text { Primarily CSF, which is a hydraulic link } \\
\text { between arteries and veins and is the path } \\
\text { for AC power. Solves the problem of } \\
\text { linking anatomically complex arteries and } \\
\text { veins in cranium. }\end{array}$ \\
\hline $\begin{array}{l}\text { Opposing } \\
\text { (circulation) } \\
\text { motion }\end{array}$ & $\begin{array}{l}\text { Motion of } \\
\text { absorber } \\
\text { mass }\end{array}$ & $\begin{array}{l}\text { Circulation } \\
\text { current }\end{array}$ & $\begin{array}{l}\text { Circulation } \\
\text { (windkessel) } \\
\text { flow }\end{array}$ & $\begin{array}{l}\text { Synchronized motion of tissues and fluids } \\
\text { in cranium that continuously oppose and } \\
\text { cancel capillary AC flow. Circulation flow } \\
\text { is the cornerstone of the cerebral } \\
\text { windkessel. }\end{array}$ \\
\hline
\end{tabular}

Fig 3 : Windkessel circuit model. The model clarifies the dynamics of the cerebral windkessel. Suppression of the AC current from the capillary path is accomplished by cyclic loading and unloading of the 'arterial' and 'venous' capacitor plates, which continuously opposes and blocks the cyclic current from the AC arterial source. The tank circuit has a DC and AC source (corresponding in the cranium to MAP and pulse pressure, respectively), a series resistor $\boldsymbol{R}_{\text {cap }}$ (corresponding to the capillary path), and a parallel RLC circuit (corresponding to the CSF, interstitial fluid, brain parenchyma, and intracranial arteries and veins, which for brevity we call the CSF path). Voltage represents pressure, current represents flow, and charge represents volume. Voltage 
across the source is a proxy for ABP, and voltage across the capillary resistor is a proxy for ICP. Capacitance $C$ represents the compliance of the extracapillary (parenchymal and CSF) path and corresponds primarily to the compliance of the intracranial veins.

Inductance $L$ represents intracranial inertance, which corresponds to the inertance of the extracapillary cranial contents. $\boldsymbol{R}_{C S F}$ represents structural damping of the vascular walls and brain parenchyma and resistance to compression/expansion of the cerebral arteries and veins. $R_{C S F}$ is effectively a venous pump. $\boldsymbol{R}_{c a p}$ corresponds to intracapillary resistance to longitudinal blood flow, and $R_{C S F}$ is the net resistance in the extracapillary space to radial expansion and relaxation of capillary walls. Note that $\boldsymbol{R}_{C S F}$ does not represent the resistance to CSF absorption. The DC power that drives CSF formation and absorption is several orders of magnitude smaller than AC power ${ }^{1}$ and is represented in this model as a miniscule component of DC power.

Cerebral windkessel impedance and effectiveness

The impedance of the cranium to cerebral blood flow is the ratio of pressure to flow, which by linear lumped-parameter approximations of intracranial dynamics is expressed according to

$$
Z_{\text {cranium }}=R_{c a p}+\frac{R_{C S F}}{\omega^{2} R_{C S F}^{2} C^{2}+\left(\omega^{2} L C-1\right)^{2}}+j\left[\frac{\omega C\left(-\omega^{2} L^{2}+\frac{L}{C}-R_{C S F}^{2}\right)}{\omega^{2} R_{C S F}^{2} C^{2}+\left(\omega^{2} L C-1\right)^{2}}\right]
$$

where $Z_{\text {cranium }}$ is the combined impedance of the capillary and CSF path, $R_{\text {cap }}$ is the resistance to longitudinal flow in the capillary lumina, $R_{C S F}$ is resistance to radial oscillations in the brain, 
CSF path, and vasculature, $\omega$ is the radial frequency expressed in radians, $C$ is capacitance, $L$ is inductance (see Table 1 for a detailed correspondence between mechanical, electrical and windkessel parameters), and $j=\sqrt{-1}$.

We designate the real and imaginary components of the windkessel impedance as $R_{w k}$ and $X_{C S F}:$

$$
\begin{gathered}
R_{w k}=\frac{R_{C S F}}{\omega^{2} R_{C S F}{ }^{2} C^{2}+\left(\omega^{2} L C-1\right)^{2}} \\
X_{C S F}=\frac{\omega C\left(-\omega^{2} L^{2}+\frac{L}{C}-R_{C S F}^{2}\right)}{\omega^{2} R_{C S F}^{2} C^{2}+\left(\omega^{2} L C-1\right)^{2}}
\end{gathered}
$$

so that

$$
Z_{\text {cranium }}=R_{\text {cap }}+R_{w k}+j X_{C S F}
$$

and

$$
Z_{w k}=R_{w k}+j X_{C S F}
$$

where $Z_{w k}$ is the impedance of the windkessel (tank part of the circuit). The windkessel impedance phase $\theta_{w k}$ is

$$
\theta_{w k}=\arctan \left(\frac{X_{C S F}}{R_{w k}}\right)
$$

and thus

$$
\theta_{w k}=\arctan \left[\frac{\omega C\left(-\omega^{2} L^{2}+\frac{L}{C}-R_{C S F}^{2}\right)}{R_{C S F}}\right]
$$

The maximal (optimal) windkessel impedance $Z_{w k}$ is the real part $R_{w k}$ when the imaginary part $X_{C S F}$ is zero. The resistance $R_{w k}$ is then a local maximum and represents 'pure' resistance without reactance. We choose pure resistance without reactance as the normal windkessel impedance because, in normal dynamics, there is minimal reflectance of the arterial pulse from 
the periphery back to the heart ${ }^{2}$, and this corresponds mathematically to resistance without reactance. With pure windkessel resistance, the optimal heart rate $\omega_{w k}$ (in radians) is

$$
\omega_{w k}=\sqrt{\frac{1}{L C}-\frac{R_{C S F}^{2}}{L^{2}}}
$$

Replacing $\omega$ in the windkessel resistance $R_{w k}$ (equation 2) with the optimal windkessel heart rate $\omega_{w k}$ (equation 8 ) we obtain the windkessel effectiveness $W_{\text {eff }}$ (i.e., the real part of the impedance offered by the tuned windkessel):

$$
W_{\text {eff }}=\frac{L}{R_{C S F} C}
$$

Windkessel effectiveness expresses the effectiveness of the optimally tuned windkessel when intracranial inertance, resistance, and compliance are taken into account.

Windkessel impedance is heart rate sensitive, and optimal dynamics correspond to specific values of $L, C, R_{C S F}$ and heart rate. When the windkessel is optimally tuned, it offers maximal impedance to AC power through the capillary path, and the magnitude of this maximal impedance is given by the windkessel effectiveness $W_{\text {eff }}$. DC power is unaffected by windkessel reactance, which only opposes accelerated flow. This selective capillary admittance to $\mathrm{DC}$ flow and impedance to AC flow is the basis for the cerebral windkessel.

\section{Data collection and analysis}

For quantitative validation of this model, we use data from a series of dogs $(18-26 \mathrm{~kg})$ previously reported [6]. Our protocol was approved by our Institutional Animal care and Use Committee (Wagshul 2007-1504 IACUC), and the animals were treated humanely in accordance with the "Guide for the care and use of laboratory animals" published by the 
National Institutes of Health. Each dog was sedated with thiopental, intubated, and anesthetized with 1.5\% isoflurane. A Microtip pressure transducer (SPR-524, 1-mm diameter, Millar Instruments) was inserted into the right common carotid artery by open cut-down and advanced rostral in the artery to just below the skull base and secured. A burr hole was drilled in the right frontal region and the dura was opened, and a second Microtip catheter was inserted 1-2 cm into the right frontal parenchyma. Data was collected for approximately 15 minutes in the resting state when ICP was normal, prior to manipulation of the ICP. All the data analyzed in this paper is data from dogs with normal resting ICP.

\section{Data analysis: data preprocessing}

As mentioned above, the data sets only contain the raw ABP and ICP time series, and their time stamps. For each data set, we estimate the real-time heart rate in a moving window. In each window, we detect the peaks of systolic and diastolic arterial pressures and their corresponding time stamps. The average heart rate in this window is calculated by counting the pairs of systolic and diastolic peaks and normalizing it by time length. Except for computing the heart rate, we filter the raw data because we are analyzing the fundamental frequency band and we want to avoid aliasing, so we apply a lowpass filter and a band-stop filter on each raw dataset. The lowpass filter is used for removing high-frequency noise and the band-stop filter for removing the respiratory effect which is usually located around $0.1 \sim 0.5 \mathrm{~Hz}$. For implementation we choose Butterworth filters.

\section{Circuit Analysis: Transfer Function}


We compare the circuit model to the model obtained by the nonparametric transfer function obtained from dog data. The transfer function is defined as the ratio of the Laplace transform of the output (the ICP, i.e., voltage across the resistor) to the Laplace transform of input (the ABP, i.e., voltage of the sources). Given the impedances of the circuit elements $R_{\text {cap }}, R_{C S F}, s L$ and $1 / s C$, the transfer function of the windkessel circuit is

$$
\begin{aligned}
H(s)=\frac{V_{o}(s)}{V_{i}(s)} & =\frac{R_{c a p}}{R_{c a p}+\frac{\left.(1 / s C)\left(R_{C S F}+s L\right)\right)}{(1 / s C)+\left(R_{C S F}+s L\right)}} \\
& =\frac{R_{c a p} L C s^{2}+R_{c a p} R_{C S F} C s+R_{c a p}}{R_{c a p} L C s^{2}+\left(R_{c a p} R_{C S F} C+L\right) s+R_{c a p}+R_{C S F}}
\end{aligned}
$$

The dynamics of pressure and flow in the windkessel can be described by second-order differential equations. To facilitate discrete time-data analysis, we map the transfer function from the Laplace domain to the $Z$ domain. We use the Tustin (bilinear) approximation [16]

$$
\begin{gathered}
z=e^{s T} \approx \frac{1+\frac{s T}{2}}{1-\frac{s T}{2}} \\
s \approx \frac{2}{T} \times \frac{z-1}{z+1}
\end{gathered}
$$

where $T$ is the sampling interval. The z-domain transfer function of the windkessel circuit is

$$
H_{c}(z) \approx \frac{N_{0}+N_{1} z^{-1}+N_{2} z^{-2}}{D_{0}+D_{1} z^{-1}+D_{2} z^{-2}}
$$

where the coefficients are

$$
\begin{gathered}
N_{0}=4 R_{c a p} L C+2 T R_{c a p} R_{C S F} C+T^{2} R_{c a p} \\
N_{1}=2 T^{2} R_{c a p}-8 R_{c a p} L C \\
N_{2}=4 R_{c a p} L C-2 T R_{c a p} R_{C S F} C+T^{2} R_{c a p} \\
D_{0}=4 R_{c a p} L C+2 T R_{c a p} R_{C S F} C+T^{2}\left(R_{c a p}+R_{C S F}\right)+2 T L \\
D_{1}=2 T^{2}\left(R_{c a p}+R_{C S F}\right)-8 R_{c a p} L C
\end{gathered}
$$




$$
D_{2}=4 R_{c a p} L C-2 T R_{c a p} R_{C S F} C+T^{2}\left(R_{c a p}+R_{C S F}\right)-2 T L
$$

\section{Time Series Modeling and Transfer Function}

Both input ABP and output ICP are time series data. We model the cerebral windkessel in the time domain by a linear autoregression of the ICP with an exogenous input (IBP), thus creating an ARX model. The general formulation of an ARX model with single input and single output is

$$
y[n]=\sum_{i=1}^{p} a_{i} y[n-i]+\sum_{j=0}^{q} b_{j} x[n-j]+\varepsilon[n]
$$

where $p$ and $q$ are the orders of the model. The symbols $a_{i}, i=1,2,3 \ldots, p$ and $b_{j}, j=0,1,2 \ldots, q$ are the coefficients of the model, and $\varepsilon[n]$ is a disturbance that represents the portion of the ICP that cannot be described by the model. The z-domain transfer function is:

$$
H(z)=\frac{b_{0}+b_{1} z^{-1}+\cdots+b_{q} z^{-q}}{1-a_{1} z^{-1}-\cdots-a_{p} z^{-p}}
$$

When the order $p=2$ and $q=2$, the transfer function is simplified to

$$
H_{A R X}(z)=\frac{b_{0}+b_{1} z^{-1}+b_{2} z^{-2}}{1-a_{1} z^{-1}-a_{2} z^{-2}}
$$

\section{Relationship between Electrical Circuit Model and Time Series Model}

Comparing equation (13) and equation (17), the transfer function of the ARX model has the same form as the transfer function of the proposed circuit model. We connect them and estimate the circuit elements by time series analysis. Ideally, the coefficients should satisfy

$$
\begin{aligned}
& -a_{1}=D_{1} / D_{0} \\
& -a_{2}=D_{2} / D_{0}
\end{aligned}
$$




$$
\begin{aligned}
& b_{0}=N_{0} / D_{0} \\
& b_{1}=N_{1} / D_{0} \\
& b_{2}=N_{2} / D_{0}
\end{aligned}
$$

Note that the coefficients $a_{i}, i=1,2$ and $b_{j}, j=0,1,2$ in equation (17) can take any values. However, the coefficients $N_{i}, i=0,1,2$ and $D_{j}, j=0,1,2$ in equation (13) have to satisfy the relationships in equation (14). Obviously, the transfer function of the circuit is a subset of the transfer functions of the ARX model. In equation (18), the mapping from the right side (circuit elements) to the left side (coefficients of the ARX model) is one-to-one. But given a set of ARX coefficients, it is not always possible to find the corresponding set of circuit elements which satisfy these equations. We discuss how to obtain a solution in the following section.

\section{Estimating the Circuit Elements according to Time-series Model}

As mentioned above, we cannot obtain the estimates of the circuit elements from the time series

model directly. Considering that the windkessel notch appears around the heart rate, in order to compare and analyze the transfer functions in a specific frequency band, we derive the mathematical expressions of the transfer functions in the frequency domain. Given equation (13), the transfer function in the frequency domain is given by

$$
H_{c}\left(e^{j \omega}\right)=\frac{N_{0}+N_{1} e^{-j \omega}+N_{2} e^{-j 2 \omega}}{D_{0}+D_{1} e^{-j \omega}+D_{2} e^{-j 2 \omega}}
$$

Applying Euler's formula $e^{j \omega}=\cos (\omega)+j \sin (\omega)$, we can rewrite the transfer function with separate real and imaginary parts:

$$
H_{c}\left(e^{j \omega}\right)=\frac{N_{r} D_{r}+N_{i} D_{i}}{D_{r}^{2}+D_{i}^{2}}+j \frac{N_{i} D_{r}-N_{r} D_{i}}{D_{r}^{2}+D_{i}^{2}}
$$

where

$$
N_{r}=N_{0} \cos (2 \omega)+N_{1} \cos (\omega)+N_{2}
$$




$$
\begin{gathered}
N_{i}=N_{0} \sin (2 \omega)+N_{1} \sin (\omega) \\
D_{r}=D_{0} \cos (2 \omega)+D_{1} \cos (\omega)+D_{2} \\
D_{i}=D_{0} \sin (2 \omega)+D_{1} \sin (\omega)
\end{gathered}
$$

Similarly, the transfer function of the ARX model can also be rewritten as

$$
H_{A R X}\left(e^{j \omega}\right)=\frac{B_{r} A_{r}+B_{i} A_{i}}{A_{r}^{2}+A_{i}^{2}}+j \frac{B_{i} A_{r}-B_{r} A_{i}}{A_{r}^{2}+A_{i}^{2}}
$$

where

$$
\begin{gathered}
B_{r}=b_{0} \cos (2 \omega)+b_{1} \cos (\omega)+b_{2} \\
B_{i}=b_{0} \sin (2 \omega)+b_{1} \sin (\omega) \\
A_{r}=\cos (2 \omega)-a_{1} \cos (\omega)-a_{2} \\
A_{i}=\sin (2 \omega)-a_{1} \sin (\omega)
\end{gathered}
$$

Then we compare their real and imaginary parts separately, and define a cost function by:

$$
\begin{aligned}
\mathcal{J}(\theta)=\sum_{\omega}\{[ & \left.\mathcal{R} e\left(H_{c}\left(e^{j \omega}\right)\right)-\mathcal{R e}\left(H_{A R X}\left(e^{j \omega}\right)\right)\right]^{2} \\
+ & {\left.\left[J_{m}\left(H_{c}\left(e^{j \omega}\right)\right)-\mathcal{J} m\left(H_{A R X}\left(e^{j \omega}\right)\right)\right]^{2}\right\} }
\end{aligned}
$$

where the unknown vector $\boldsymbol{\theta}$ contains the circuit elements $R_{\text {cap }}, R_{C S F}, L$ and $C$. The cost function indicates the sum of squared distances between the real parts and imaginary parts of the transfer functions from the circuit and the ARX model at each frequency. We minimize $\mathcal{J}(\boldsymbol{\theta})$ over the frequency band containing the heart rate and under the constraints $\boldsymbol{\theta}>\mathbf{0}$. 


\section{Results}

\section{Simulation of circulation flow in tank circuit}

To verify the tank circuit model, we simulate the circulation flow by using a group of known circuit elements and an input voltage source. Estimation of model parameters of the cerebral windkessel based on physiological values is limited by the lack of experimental data on which to base parameter values (please see Limitations of our study below for a full discussion of our selection of parameter values). We set the circuit elements values at $R_{\text {cap }}=1 \mathrm{ohm}, R_{C S F}=$ $5 \mathrm{ohm}, L=1$ henry and $C=0.01 \mathrm{farad}$, and the input voltage is a sinusoidal signal with DC level $60 \mathrm{mV}$. The charge and discharge of the capacitor is shown in Fig 4, where the variations of charges on positive plate and negative plate represent expansion and relaxation of arteries and veins. In Fig 5A we show the phase measured experimentally in dogs, and in Fig 5B we show input and output voltages of the model to show phase relationships. The leading ICP phase of the circuit model correlates with the leading ICP phase in the experimental dog model, and this suggests that the counterintuitive phase lead of the ICP with respect to the ABP in the experimental animals is due to the dynamics inherent to pulsation absorption in a damped system.

Several investigators have noted that in normal dynamics the ICP pulse precedes the carotid arterial pressure pulse by about one-sixth of the cardiac cycle [6]. This is, of course, counterintuitive. Windkessel theory shows that in a windkessel mechanism with resistance, optimal pulsation suppression occurs when the frequency of pulsations is equal to the antiresonant frequency of the windkessel, which is the frequency at which the reactance (the imaginary part of the windkessel impedance) is zero and the real part is maximal (Equations 1 and 8). If we assume that the intracranial windkessel is more damped (due to the structural 
damping of the brain parenchyma) than the extracranial arterial windkessel, Equation 8 implies that the cerebral windkessel will have an inertial bias compared with the extracranial arterial windkessel, causing a leading ICP waveform with respect to the ABP waveform. The windkessel model reproduces the observed phase lead of ICP with respect to ABP and explains it as corresponding to the optimization of the cerebral windkessel when the damping of the cranium exceeds the damping of the extracranial arterial tree, which is the normal state.

Fig 4: Circulation flow of the cerebral windkessel and suppression of capillary AC power Suppression of the arterial pulse in the capillaries is accomplished in the cranium by a cyclic exchange of volume between the intracranial arteries and veins, linked by the CSF. This is analogous to the circulation current in a tank circuit, which represents the same dynamics. At every moment through the cardiac cycle, the flow of volume in the cerebral windkessel (the extracapillary cranial contents) is equal to and opposite the arterial pulse entering the capillary bed, which cancels the pulse in the arterial inflow to the capillaries and diverts it through the CSF to the intracranial veins. This cyclic exchange of volume between the intracranial arteries and veins can be simulated in the tank circuit as the loading and unloading of charge on the 'arterial' and 'venous' plates of the capacitor in the tank (Fig 3), and it renders capillary blood flow (nearly) pulseless.

Fig 5A and 5B: The phase lead of ICP with respect to ABP in the cerebral windkessel. Fig 5A shows the experimentally measured ICP lead with respect to the arterial pulse in a dog from Wagshul et al [6]. Fig 5B shows the lead of the voltage across the main resistor (which is the ICP proxy) in the tank circuit model and the voltage across the source (which is the ABP proxy). In Fig 5B, the solid line is the ABP and the dotted line is the ICP, as in Fig 5A. 


\section{Model evaluation: ICP prediction by ARX model}

We evaluate the time-series model by calculating the relative errors of ICP prediction. The 10second windows are used for analyzing the segments of processed ABP and ICP. We obtain the estimated coefficients $a_{i}, i=1,2$ and $b_{j}, j=0,1,2$ by fitting segments in the model and predict the ICP at the next time instant according to the coefficients. Table 2 summarizes the average relative prediction error for each dog data set.

Table 2 Average relative error of ICP prediction using ARX model.

\begin{tabular}{|c|c|c|c|}
\hline Data sets & $\begin{array}{c}\text { Average relative } \\
\text { error (\%) }\end{array}$ & Data sets & $\begin{array}{c}\text { Average relative } \\
\text { error (\%) }\end{array}$ \\
\hline No. 1 & 0.48 & No. 7 & 2.75 \\
\hline No. 2 & 1.81 & No. 8 & 0.50 \\
\hline No. 3 & 0.16 & No. 9 & 2.10 \\
\hline No. 4 & 0.48 & No. 10 & 0.67 \\
\hline No. 5 & 0.94 & No. 11 & 1.31 \\
\hline No. 6 & 1.14 & No. 12 & 0.75 \\
\hline
\end{tabular}

Model evaluation: circuit elements estimation and transfer function comparison

Given the estimated coefficients of the ARX model, we correlate the proposed circuit model with the ARX model and estimate the circuit elements by using the method shown above. The 10second segments of ABP and ICP from dog data sets are selected for testing. Among 12 dogs, we select a total of 16 segments from 4 dogs and summarize their parameters and estimate the circuit elements in Table 3. We plot and compare the gain of the transfer function from the Fast 
Fourier Transform (FFT), ARX and circuit models according to the estimated circuit elements. The similarity between the transfer functions of the model and the actual data from the dogs is shown in Fig 6.

Table 3: The parameters of dog data and corresponding estimation results of windkessel circuit.

Four 10-second segments from Dog No.3, No.5, No.10 and No.12 are selected for testing. The $3^{\text {rd }}$ and $5^{\text {th }}$ dogs have relatively large mean ICPs, and the $10^{\text {th }}$ and $12^{\text {th }}$ dogs have small mean ICPs. Both ICP's represent normal baseline dynamics in the animals. In Fig 6, we plot the transfer functions of the circuit according to the results of Dog No. 12 shown in the table.

\begin{tabular}{|l|l|l|l|l|l|l|c|}
\hline Data set & $\begin{array}{l}\text { Segments } \\
(\mathrm{s})\end{array}$ & $\begin{array}{l}\text { Heart } \\
\text { rate } \\
(\mathrm{Hz})\end{array}$ & $\begin{array}{l}\text { Mean } \\
\text { ICP } \\
(\mathrm{mm} \\
\mathrm{Hg})\end{array}$ & $R_{\text {cap }}$ & $R_{\text {CSF }}$ & $L$ & $C$ \\
\hline Dog 3 & $640-650$ & 0.60 & 23.71 & 0.6959 & 6.4382 & 5.1452 & 0.0119 \\
\hline Dog 3 & $690-700$ & 0.76 & 23.39 & 1.0012 & 5.3729 & 3.7022 & 0.0102 \\
\hline Dog 3 & $740-750$ & 0.91 & 22.63 & 0.6659 & 7.9382 & 3.0083 & 0.0090 \\
\hline Dog 3 & $950-960$ & 0.61 & 21.63 & 0.3049 & 5.7786 & 2.6780 & 0.0250 \\
\hline Dog 5 & $260-270$ & 0.89 & 23.07 & 3.0743 & 5.2010 & 2.2801 & 0.0138 \\
\hline Dog 5 & $410-420$ & 0.89 & 22.70 & 0.5493 & 1.6838 & 0.5819 & 0.0890 \\
\hline Dog 5 & $500-510$ & 0.69 & 22.66 & 1.4190 & 2.6838 & 1.2882 & 0.0322 \\
\hline Dog 5 & $570-580$ & 0.69 & 22.77 & 1.0802 & 2.3482 & 1.3001 & 0.0621 \\
\hline Dog 10 & $130-140$ & 0.50 & 6.5 & 0.9010 & 8.0352 & 4.0335 & 0.0195 \\
\hline Dog 10 & $180-190$ & 0.50 & 5.9 & 0.5624 & 6.0352 & 5.6320 & 0.0136 \\
\hline
\end{tabular}




\begin{tabular}{|l|l|l|l|l|l|l|l|}
\hline Dog 10 & $250-260$ & 0.63 & 5.5 & 0.5630 & 6.1832 & 3.8210 & 0.0199 \\
\hline Dog 10 & $350-360$ & 0.87 & 4.9 & 0.8218 & 9.3989 & 2.2089 & 0.0129 \\
\hline Dog 12 & $230-240$ & 0.98 & 5.6 & 0.3760 & 6.7054 & 5.3270 & 0.0114 \\
\hline Dog 12 & $260-270$ & 0.98 & 6.2 & 0.5102 & 6.4355 & 3.4145 & 0.0088 \\
\hline Dog 12 & $310-320$ & 0.98 & 6.9 & 0.7790 & 8.1562 & 4.5355 & 0.0060 \\
\hline Dog 12 & $360-370$ & 0.98 & 8.6 & 0.9495 & 8.0346 & 4.7178 & 0.0051 \\
\hline
\end{tabular}

Fig 6: The comparison of the transfer functions of the windkessel model and the dog data. Given the dog data, the transfer function from the output ICP to input ABP can be obtained directly by computing the FFT. According to the model, the transfer functions of the ARX and circuit models should capture the salient features of the transfer function from the dog data. The transfer function of the circuit should be approximately equivalent to the ARX model near the heart rate frequency. In this figure, we show results of four examples with normal mean ICP.

\section{Discussion}

Our model points to a specific understanding of the cerebral windkessel. In systole, caudal motion of the brain parenchyma opposes cranial motion of the arterial pulse entering at the skull base. Systolic centrifugal motion of the parenchyma opposes centripetal inflow of the arterial pulse in the perforating arteries, and radial inward compression by the expanding brain parenchyma opposes radial outward expansion of the capillary walls [2]. 
In diastole, cranial rebound of the brain opposes diastolic caudal regress of the arterial pulse at the skull base. Diastolic centripetal motion of the cortex opposes diastolic centrifugal regress of the arterial pulse in the perforating arteries, and centrifugal relaxation of the parenchyma opposes centripetal relaxation of the capillary walls [2].

This cyclic exchange of volume between arterial and venous expansion and relaxation is analogous to the exchange of charge between the 'arterial' and 'venous' capacitor plates in the circuit. This is the circulation current, which is the basis for the cerebral windkessel. This continuous balance of volume exchange suppresses radial motion of the capillary walls. This reciprocal vascular, CSF and brain motion has been visualized on flow MRI [17]. In our study, ARX modeling of the transfer function of the tank circuit and of the ABP and ICP waveforms in dogs shows that the dynamics of the circuit and the cerebral windkessel are fundamentally the same.

\section{Correspondence between the band-stop windkessel model and empirical characteristics of the cerebral windkessel}

As noted, our model correlates to a high degree with experimental data from dogs. The close correspondence between our model and experimental data is surprising-the wave trap circuit is a simple linear lumped parameter two-degree of freedom electrical system, whereas the cerebral windkessel is implemented in an exceedingly complex non-linear distributed parameter biological system. The close correspondence suggests that the basic dynamics of the cerebral windkessel are similar to the dynamics of a simple anti-resonant band-stop filter.

In the Introduction, we noted several characteristics of intracranial dynamics and the cerebral windkessel that are inconsistent with traditional pressure-volume models. The band-stop windkessel model is consistent with each of these characteristics. 
1. The band-stop model quantitatively replicates the ABP-ICP transfer function of the physiological data with high accuracy, and the stop band in the frequency domain corresponds to the normal local minimum of ICP amplitude observed experimentally and clinically in the time domain. The band-stop filter suppresses the high powerdensity band of ABP near the heart rate and thus provides optimal protection of the microcirculation from arterial pulsatility, which is the fundamental feature of the windkessel.

2. The observed phase transition near the central frequency of the stop band [6] is replicated in the band-stop model as a natural consequence of the anti-resonant mechanism that underlies the pulsation suppression.

3. The phase lead of ICP with respect to ABP in normal dynamics [Fig $5 A$ ] is inexplicable by pressure-volume models. However, in the steady state of the proposed circuit, individual harmonics of the output (ICP) may lead or lag corresponding harmonics of the input $(A B P)$ in accordance with the resistance and reactance of the system. The phase lead of the ICP with respect to the ABP is explained in the band-stop filter model of the windkessel as a consequence of physiological optimization of pulsation suppression in the arterial and intracranial pathways, which have different degrees of damping (see Fig 7 for a detailed explanation and simulation of phase lead).

4. With pathological changes in intracranial dynamics, the windkessel may be impaired, which in the band-stop filter model corresponds to attenuation of the notch band in the frequency domain. Traditional pressure-volume models, lacking resonant properties, cannot simulate band-stop dynamics.

5. In the time domain, the amplitude of the intracranial pressure (ICP) pulse (the ICP difference from diastole to systole) is normally at a minimum-either lowering or raising ICP increases the ICP pulse amplitude. This is inexplicable in a pressure- 
volume model, in which the ICP pulse pressure should diminish with low ICP (high compliance) and increase with high ICP (low compliance). In the band-stop model this local minimum of ICP pulse pressure is explained naturally as a consequence of impairment of the windkessel (with greater transmission of the ABP pulse to the parenchyma) due to excessively high or excessively low intracranial compliance. Pressure-volume models, lacking resonant properties, cannot explain this bi-phasic response of the ICP pulse amplitude to mean variations in mean ICP and in intracranial compliance.

6. In the cranium, the windkessel splits $D C$ and $A C$ power of $C B F$ into two different paths-DC power flows in the capillaries and AC power flows in the CSF spaces. The low impedance of the CSF spaces to AC power accounts for the normal minimal retrograde reflectance of the arterial pulse retrograde through the arterial tree ${ }^{12}$.

\section{How is the cerebral windkessel implemented anatomically and physiologically in the cranium?}

Based on the close correspondence between the transfer function of $\mathrm{AC}$ suppression in the circuit and of arterial pulse suppression in the cranium, we propose that the tank circuit model provides insight into the physical basis for the windkessel in the cranium.

In an electrical band-stop filter (i.e., a tank circuit) the suppression of frequencies in the stop band is accomplished by a circulation current between the inductor and the capacitor that continuously opposes the current in the main line from the alternating current (AC) source [Fig 7]. The circulation $A C$ current in an electrical anti-resonant band-stop filter is the reciprocating storage and release of kinetic and potential energy in circuit components. 
Circulation current oscillates back and forth in synchrony with the AC source current and cancels the source current [Fig 7]. If we consider the AC cycle as systole and diastole and define AC flow in the main (capillary) resistor away from the source as cranial and toward the source as caudal, in systole the AC source current flows cranially while the circulation current from the tank flows caudally. In diastole, the source current flows caudally while the circulation current from the tank flows cranially. The AC source current and the circulation current are equal in magnitude and opposite in direction and meet in the main (capillary) path, where the currents cancel each other [Fig 7]. The cancellation is frequency-selective- only AC source current at the anti-resonant frequency of the circulation current is cancelled. Anti-resonance corresponds to maximal impedance, which corresponds to a specific balance of frequency, inductance, resistance, and capacitance in the tank circuit. Note that DC power passes through the main (capillary) path unimpeded by the circulation current because DC flow is insensitive to frequency and the reactance of the tank circuit only opposes AC (i.e., accelerated) flow. In other words, the tank circuit selectively passes DC power and impedes AC power.

Fig 7: Windkessel circuit model in systole and diastole

The tank circuit has a DC and AC source (corresponding in the cranium to MAP and pulse pressure, respectively), a series resistor $R_{\text {cap }}$ (corresponding to the capillary path), and a parallel RLC circuit (corresponding to the CSF, interstitial fluid, brain parenchyma, and intracranial arteries and veins, which for brevity we call the CSF path). Voltage represents pressure, current represents flow, and charge represents volume. Voltage across the source is a proxy for $\mathrm{ABP}$, and voltage across the capillary resistor is a proxy for ICP. Capacitance $C$ represents the compliance of the extracapillary (parenchymal and CSF) path and corresponds primarily to the compliance of the 
intracranial veins. Inductance $L$ represents intracranial inertance, which corresponds to the inertance of the extracapillary cranial contents. $R_{C S F}$ represents structural damping of the vascular walls and brain parenchyma and resistance to compression/expansion of the cerebral arteries and veins. $R_{C S F}$ is effectively a venous pump. $R_{c a p}$ corresponds to intracapillary resistance to longitudinal blood flow, and $R_{C S F}$ is the net resistance in the extracapillary space to radial expansion and relaxation of capillary walls. Note that $R_{C S F}$ does not represent the resistance to CSF absorption. The DC power that drives CSF formation and absorption is several orders of magnitude smaller than AC power [1] and is represented in this model as a miniscule component of DC power.

The model clarifies the dynamics of the cerebral windkessel. Suppression of the AC current from the capillary path is accomplished by cyclic loading and unloading of the 'arterial' and 'venous' capacitor plates, which continuously opposes and blocks the cyclic current from the AC arterial source. This circulation current is the motion of the brain and CSF in the cardiac cycle-in systole the brain expands and moves caudally, and in diastole it relaxes and rebounds cranially [17]. This accomplishes the cerebral windkessel.

In the cranium, the current is the motion of intracranial blood, CSF and brain tissue, and the circulation current is the reciprocating storage and release of kinetic energy in the motion of blood, CSF and brain tissue and potential energy in the compliance of the cranial contents. [Fig 8]

Fig 8: Implementation of the cerebral windkessel by cyclic brain, CSF, and vascular motion during the cardiac cycle. 
8A) Using flow MRI imaging, Greitz ${ }^{17}$ has shown that during systole the brain undergoes two motions: centrifugal expansion and caudal displacement toward the skull base. During diastole, the brain relaxes centripetally, and rebounds cranially. This motion is represented in 4 panels, a-d (from Greitz et al. [17] with permission).

8B) Motion of the brain in systole. In systole, the arterial pulse enters the cranium via the circle of Willis at the skull base and enters the brain via perforating arteries that enter the parenchyma at right angles to the cortical surface. Note that the motion of the brain during systole continuously opposes the systolic pulsatile bolus of arterial blood. The systolic arterial bolus is opposed by simultaneous downward displacement of the brain against the arterial expansion in the circle of Willis, and centrifugal expansion of the brain opposes the arterial bolus entering via the perforating arteries entering the cortex.

The systolic arterial pulse is depicted by red solid arrows, and the centrifugal and caudal brain motion is depicted by partially open arrows. In systole, brain expansion and caudal brain displacement deflects a portion of the systolic arterial pulse away from the capillary circulation and diverts the pulsatile energy through the CSF spaces to the veins.

8C) Motion of the brain in diastole. The diastolic arterial pulse is depicted by red solid arrows, and the centripetal and cranial brain motion is depicted by partially open arrows. In diastole, centripetal and caudal brain relaxation opposes diastolic relaxation of the arteries, which augments the capillary circulation and returns a portion of the systolic kinetic energy to the diastolic capillary flow.

The net effect of cyclic brain expansion and relaxation coupled to arterial expansion and relaxation during the cardiac cycle is to buffer systolic capillary perfusion and enhance diastolic 
capillary perfusion, which provides nearly pulseless irrigation of the microvasculature. This cyclic opposition of brain motion to the arterial pulse is the mechanical basis for the cerebral windkessel. The elastance necessary for this process is in the elastance of the vascular walls and in the displacement of blood from the cerebral veins. The physical coupling between cyclic arterial expansion/relaxation and venous compression and refilling is provided by the CSF, which serves as the hydraulic link between the intracranial arteries and veins through which the arterial AC power (i.e. the arterial pulse) is passed to the veins, bypassing the microvasculature. Patency of the CSF spaces and unimpeded pulsatile motion of CSF is essential for normal function of the cerebral windkessel.

Physiological elements in the cranium correspond to electrical elements in the circuit: heart rate to frequency, pressure to voltage, pulsatile motion of blood, brain, and CSF to AC current, intracranial inertance to inductance, intracranial damping and resistance to resistance, and intracranial compliance to capacitance.

In the same manner that the tank circuit suppresses electrical AC power and passes DC power, the cerebral windkessel suppress capillary AC power and passes capillary DC power.

What is the anatomical and physiological basis for this circulation current in the cranium? MRI imaging of pulsatile brain motion [17] shows CSF, blood, and brain motion that corresponds to this circulation current [Fig 8]. In systole, arteries and the brain parenchyma expand, veins are compressed, and the brain shifts caudally toward the skull base. In diastole, the arteries and brain relax, veins refill, and the brain rebounds cranially away from the skull base. This pulsatile circulation flow (i.e., pulsatile motion) in intracranial arteries, veins, parenchyma, and CSF 
continuously opposes and cancels pulsatile capillary flow and is the analogue of the circulation current in the tank of the electrical band-stop filter [Fig 7].

The windkessel short-circuits the AC power of the arterial pulse by passing it through the CSF to the veins, and thus, back to the heart, while the windkessel passes DC power of cerebral perfusion unimpeded through the capillaries. This is analogous to the AC suppression and DC admittance in the tuned tank circuit. Thus, the windkessel efficiently passes all DC and AC power of CBF from the left ventricle via the arteries through the cranium and via the veins back to the right atrium [2]. By splitting DC power through the capillary path, and AC power through the CSF path, the windkessel provides cerebral perfusion that is effective, efficient, and safe.

\section{Limitations of our study}

Our windkessel model has limitations characteristic of all models of complex biological systems. In addition, modeling of capillary pulsatility presents unique challenges.

1) The model is a simple linear lumped parameter simulation (the circuit) of an enormously complex non-linear distributed parameter system (the cranium). The only completely accurate model of a complex system is the system itself. However, the model provides important insight into the cerebral windkessel because it simplifies its dynamics. By reducing the distributed non-linear inertance, capacitance, and damping of the cranial contents to a single linear inductor, capacitor, and resistor, we can more readily understand the essential characteristics of the dynamics underlying the windkessel. ARX modeling quantifies our confidence that the simplification imposed by the model actually 
reveals the essential dynamics of the cerebral windkessel, and the simple lumped structure of the model shows how the brain, blood, and CSF motion revealed by flow MRI during the cardiac cycle [17] accomplishes suppression of the arterial pulse in the capillaries.

2) As noted earlier, our estimation of model parameters is limited by lack of knowledge of the values of the parameters in the cranium. It is important to realize that the damping, compliance and inertance relevant to the cerebral windkessel are the parameters specific to the radial micro-motion of capillary walls in the brain parenchyma. These values differ from the values of total cerebrovascular resistance and intracranial compliance. Windkessel damping entails damping of the radial motion of brain tissue during the cardiac cycle and is not identical with cerebrovascular resistance to smooth blood flow through the capillary bed. Windkessel compliance refers to the compliance of capillary walls encased in brain parenchyma, not to the compliance of CSF spaces, which is the compliance parameter commonly measured by the pressure volume index. Inertance in our model refers to the pressure change necessary to cause a one-unit change in volumetric flow. In our model, flow is the velocity of radial motion of capillary walls, which has never been measured in the cranium.

Given these constraints, we have chosen model parameters of resistance, capacitance, and inductance based on four considerations:

1) We set parameters to correlate with values of resistance, capacitance and inertance measured in the aorta by Stergiopulos et. al. [5]

2) We set the frequency to correspond with the heart rate.

3) We set the ratio of $R_{c a p}$ and $R_{c s f}$ to correspond to our empirically measured phase lead of ICP to ABP at the frequency of the heart rate. 
4) We set input and output voltages to reflect the magnitudes of $A B P$ and ICP, respectively.

The conceptual and technical difficulties inherent to experimental measurement of cerebral capillary inertance, capacitance, and damping and the importance of these parameters to the windkessel dynamics highlights the need for such research as a predicate for a deeper understanding of blood flow in the cerebral microcirculation.

The salient parameter of the windkessel at the capillary level is a specific subset of intracranial dynamics - i.e., radial displacement of the capillary wall during the cardiac cycle, which is necessarily minimal if capillary integrity, normal capillary filtration, and Starling dynamics are to be preserved. However, conventional techniques (i.e., ICP monitoring, Doppler arterial flow, flow-sensitive MRI)) do not directly measure radial capillary displacement. This is an inherent limitation of our model because we are unable to measure the parameter-capillary wall motion-that is fundamental to the cerebral windkessel.

In our study, we consider the measurement of ICP to be a proxy for cerebral capillary wall motion, because pulse pressure and flow variation during the cardiac cycle corresponds to capillary flow variation and capillary wall motion in the microvasculature. Our circuit model addresses this limitation by allowing us to measure the voltage transfer function of the circuit that corresponds to optimal pulsation suppression by the windkessel (i.e., minimal variation in charge accumulation on the capacitor), which corresponds to the ABP-ICP transfer function measured in the experimental animals. Accumulation and release of charge on the circuit capacitor is analogous to capillary expansion and relaxation, and the circuit model permits simulation of this important but difficult to measure parameter. Brain expansion and relaxation during the cardiac cycle measured by flow MRI [17] also serves as a proxy for capillary pulsatility, and thus as a proxy for 
the effectiveness of the cerebral windkessel mechanism. The minimal state of brain expansion in normal dynamics (and its increase in hydrocephalus [17]) supports the inference that disorders of intracranial dynamics such as hydrocephalus are accompanied by windkessel impairment and that windkessel impairment may be an important but unexplored aspect of the pathophysiology of disorders of intracranial dynamics.

\section{Physiological implications}

There are several important physiological implications of this understanding of the cerebral windkessel.

\section{The windkessel and energy dynamics}

The cornerstone for understanding the physiological and clinical implications of windkessel theory is based on the principle of energy conservation, which is analogous to the principle of mass conservation inherent to the Monro-Kellie doctrine. All energy of CBF entering the cranium must leave the cranium or accumulate in it. In normal dynamics, there is no net energy accumulation, but impairment of the cerebral windkessel may create dynamic instability with progressive accumulation of energy in the brain parenchyma. Pressure is energy density, so this parenchymal energy accumulation corresponds to increased intracranial pressure. This potential for inexorable energy accumulation and dynamic instability is inherent to the dynamics of band-stop filters, and it is reasonable to infer that dynamic instability of the cerebral windkessel may play a role in brain edema and increased intracranial pressure in a variety of clinical situations, such as hydrocephalus, tumor, head injury, and stroke. Windkessel theory 
provides a new means of understanding this pressure/energy accumulation and of devising novel approaches to treatment, as will be discussed below.

\section{Physiological regulation}

The windkessel is frequency-sensitive and heart rate varies considerably under normal circumstances, so continuous optimal tuning of the windkessel implies physiological regulation. The nature of this regulation is unknown, but it likely involves feedback control of intracranial inertance, compliance and/or resistance. It is noteworthy that venous compliance is the only significant source of intracranial compliance over the time course of the cardiac cycle, and cerebrovenous pressure (which correlates with compliance) is regulated by the autonomic nervous system $[18,19]$. Autonomic coupling of heart rate to cerebrovenous pressure would be a plausible mechanism for windkessel regulation.

\section{Phase of ICP}

Counterintuitively, the normal ICP pulse precedes the ABP pulse by about one sixth of the cardiac cycle [2]. The windkessel circuit model reproduces this ICP phase lead, which corresponds to an inertial bias in the intracranial windkessel as compared with the extracranial windkessel (Figs $5 \mathrm{~A}$ and $5 \mathrm{~B}$ ). The counterintuitive lead of ICP with respect to ABP is due to the impedance characteristics of an optimally tuned damped band-stop filter [2].

\section{Windkessel impairment}

Conceptually, there are two different kinds of windkessel impairment. Impairment may be due to detuning of the windkessel or to loss of tuned windkessel effectiveness. 


\section{Impairment due to detuning of the windkessel}

The tuned heart rate (i.e., the central frequency of the windkessel notch) is defined by

$$
\omega_{w k}=\sqrt{\frac{1}{L C}-\frac{R_{C S F}^{2}}{L^{2}}}
$$

If the actual heart rate does not correspond to $\omega_{w k}$, the windkessel is detuned and ineffective. The sensitivity of the windkessel to detuning can be estimated by considering the effect on $\omega_{w k}$ of a small perturbation in resistance, compliance or inertance expressed respectively by the partial derivatives

$$
\begin{gathered}
\frac{\partial \omega_{w k}}{\partial R_{C S F}}=\frac{-R_{C S F}}{\omega_{w k} L^{2}} \\
\frac{\partial \omega_{w k}}{\partial C}=\frac{-1}{2 \omega_{w k} L C^{2}} \\
\frac{\partial \omega_{w k}}{\partial L}=\frac{R_{C S F}^{2} C-\frac{L}{2}}{\omega_{w k} L^{3} C}
\end{gathered}
$$

The optimal windkessel frequency $\omega_{w k}$ decreases with increased resistance and compliance

and varies with inertance. As noted, the coupling of the heart rate to $\omega_{w k}$ when the parameters change implies physiological regulation of the windkessel.

\section{Impairment due to loss of effectiveness of the tuned windkessel.}

Even when the windkessel is optimally tuned (that is, the actual heart rate corresponds to $\omega_{w k}$ ), the windkessel effectiveness given by

$$
W_{\text {eff }}=\frac{L}{R_{C S F} C}
$$

which varies according to the partial derivatives 


$$
\begin{gathered}
\frac{\partial W_{e f f}}{\partial R_{C S F}}=\frac{-L}{R_{C S F}^{2} C} \\
\frac{\partial W_{e f f}}{\partial C}=\frac{-L}{R_{C S F} C^{2}} \\
\frac{\partial W_{e f f}}{\partial L}=\frac{1}{R_{C S F} C}
\end{gathered}
$$

The windkessel effectiveness decreases with increased resistance and compliance and increases with increased inertance. Note that the impairment of the windkessel effectiveness can occur despite optimal tuning (i.e. coupling of the heart rate to $\omega_{w k}$ ). Abnormal intracranial parameters (e.g. high resistance, high compliance or low inertance) diminish the windkessel effectiveness, even if the windkessel is optimally tuned to the heart rate.

\section{Obstructive hydrocephalus}

Obstruction of CSF spaces (i.e., decrease in effective volume of the CSF path) diminishes the cerebral windkessel effectiveness $W_{\text {eff }}$ by increasing CSF resistance $R_{C S F}$ (equation 9). If the CSF path is a cylindrical tube, the resistance $R_{C S F}$ is given by Poiseuille's Law:

$$
R_{C S F}=\frac{8 \eta l}{\pi r^{4}}
$$

where $r$ is the radius of the CSF space, $l$ is the length of the (cylindrical) space, and $\eta$ is the viscosity of the CSF. Combining equations (9) and (17), the windkessel effectiveness in a cylindrical CSF path is

$$
W_{e f f}=\frac{L \pi r^{4}}{8 \eta l C}
$$

The sensitivity of $W_{\text {eff }}$ to effective radius $r$ of the CSF path (as a proxy for obstruction) can be estimated by 


$$
\frac{\partial W_{e f f}}{\partial r}=\frac{L \pi r^{3}}{2 \eta l C}
$$

Windkessel effectiveness varies with the cubic power of the effective radius of the CSF space. That is, ventricular obstruction impairs windkessel effectiveness, and ventriculomegaly improves windkessel effectiveness. In windkessel theory, ventriculomegaly is an adaptation to obstructive hydrocephalus, rather than a passive consequence of accumulation of CSF in the ventricles.

\section{Obstructive hydrocephalus in terms of impaired intracranial distribution of DC and AC power of CBF}

An equivalent perspective is that obstructive hydrocephalus is a disorder of DC and AC power

flow in the cranium. Normally, DC and AC power are mixed in arterial and venous CBF entering and leaving the cranium, but at the level of the capillaries, essentially all power of CBF is DC. AC power is routed through the CSF pathway to the veins.

Obstruction of the CSF path impedes the flow of $A C$ power and redirects $A C$ power through the capillary path, which can cause cerebral edema and catastrophic capillary disruption.

Consequential enlargement of the CSF path by ventriculomegaly partially or completely restores the extracapillary path for AC power, which restores the windkessel.

From the standpoint of intracranial power dynamics, ventriculomegaly in obstructive hydrocephalus is not a consequence of accumulation of CSF due to an imbalance of formation and absorption, but a physiological adaptation that restores the CSF path for AC power necessary for normal windkessel dynamics. 


\section{Pseudotumor cerebri and the lack of ventricular dilation}

Like obstructive hydrocephalus, pseudotumor cerebri (PTC) is associated with cerebrovenous hypertension, increased resistance to CSF absorption, and increased intracranial pressure [20]. Unlike obstructive hydrocephalus, the CSF spaces in PTC are not obstructed, and PTC is not characterized by ventriculomegaly.

Windkessel theory offers an explanation for the lack of ventricular dilation in PTC. PTC decreases intracranial compliance (due to cerebrovenous hypertension), which, in the absence of CSF path obstruction, increases windkessel effectiveness $W_{\text {eff }}$ (equation 9). In this way, PTC differs from obstructive hydrocephalus in that in PTC there is no windkessel impairment. In terms of power, PTC is a disorder of DC power (high DC pressure in the capillaries), but not AC power (normal CSF path resistance maintains the windkessel). PTC, unlike hydrocephalus, would not be ameliorated by ventriculomegaly.

Flow MRI [17] has shown abnormally large systolic brain expansion in hydrocephalus and normal (minimal) systolic brain expansion in pseudotumor cerebri, which is consistent with the view that the windkessel is impaired in hydrocephalus but preserved in PTC.

\section{Low pressure hydrocephalus}

Low pressure hydrocephalus is an unusual and perplexing syndrome in which patients who are shunted for hydrocephalus present in deep coma with bradycardia and massive ventricular dilation. Intracranial pressure is paradoxically quite low. This state has been attributed to excessively low brain turgor (excessively high compliance) of unknown etiology [21]. Two therapeutic approaches are effective in reversing the state: 
1) external ventricular drainage for at least several days at a very low level to create siphoning

2) compression of the jugular veins by neck wrapping

After recovery, it is imperative that patients have shunt valves that siphon. Placement of antisiphon devices tends to cause recurrence of the syndrome.

Windkessel theory provides a hypothesis for these dynamics. Consideration of the equation (9) for windkessel effectiveness

$$
W_{e f f}=\frac{L}{R_{C S F} C}
$$

suggests (as noted above) that windkessel impairment in obstructive hydrocephalus is a consequence of increased resistance $\boldsymbol{R}_{C S F}$ in the CSF pathway. In low pressure hydrocephalus, a state of excessively high compliance $\boldsymbol{C}$ will tend to exacerbate the underlying windkessel impairment associated with obstructive hydrocephalus, locking the patient into a state of windkessel impairment with low ICP (due to excessively high intracranial compliance).

Amelioration of this state would entail ventricular dilation to reduce the resistance in the CSF space. If this physiological adaptation is insufficient, the patient is locked into intractable 
windkessel impairment due to excessively high $\boldsymbol{R}_{\boldsymbol{C S F}}$ and $\boldsymbol{C}$ with paradoxically low ICP due to the excessively high intracranial compliance.

Windkessel theory explains the effectiveness of rather unorthodox treatment methods.

Siphoning markedly decreases the resistance of the CSF pathways (see below) and jugular vein compression decreases cerebral compliance, both of which lower the denominator in the windkessel effectiveness equation (9) and thereby restore windkessel effectiveness and reverse the syndrome.

\section{CSF diversion}

In windkessel theory, CSF diversion is of value in two ways:

First, CSF diversion diverts power from the cranium by draining kinetic and potential energy carried in the CSF. ICP is intracranial energy density, and drainage of energy lowers pressure.

Second, CSF diversion increases windkessel effectiveness $W_{\text {eff }}$ by providing a parallel low resistance path for $\mathrm{AC}$ power.

Third ventriculostomy also reduces $R_{C S F}$ and increases $W_{\text {eff }}$ if the ostomy is large enough and the subarachnoid spaces are patent.

\section{Cushing's reflex}


Complete obstruction of the CSF path (i.e., infinitely large $R_{C S F}$ ) due to either severe obstructive hydrocephalus or to severe brain swelling with obliteration of CSF spaces ('ground glass brain') destroys the windkessel. Elevated ICP impairs DC capillary perfusion and complete obstruction of the CSF path redirects all AC power through the capillaries. From this perspective, Cushing's reflex-hypertension and bradycardia—has a physiological rationale [22]. Hypertension restores capillary DC power by increasing DC offset (mean arterial pressure) and bradycardia minimizes capillary AC power, because AC power is proportional to frequency.

In windkessel theory, Cushing's reflex is an accessory windkessel in extremis.

\section{New directions in the understanding of cerebral blood flow and intracranial dynamics}

Table 4 summarizes the postulated windkessel impairments in obstructive hydrocephalus, low pressure hydrocephalus, and pseudotumor cerebri, and the amelioration provided by ventriculomegaly, CSF diversion, and Cushing's reflex.

\section{Table 4 Windkessel impairment in disorders of intracranial dynamics}

Windkessel theory can explain several features of common disorders of intracranial dynamics as impairment of the tuned windkessel, as given by

$$
W_{e f f}=\frac{L}{R_{C S F} C}
$$

Where $\mathrm{W}_{\text {eff }}$ is the windkessel effectiveness (i.e., the windkessel impedance in capillaries when the windkessel is tuned to the heart rate.), $L$ is intracranial inertance, $R_{C S F}$ is resistance in the 
CSF path, and $C$ is intracranial compliance. Please see Table 1 for more detailed discussion of parameters and units.

\begin{tabular}{|c|c|c|}
\hline & $\begin{array}{l}\text { Cause of windkessel } \\
\text { impairment }\end{array}$ & $\begin{array}{l}\text { Windkessel impairment } \\
\text { expressed as disorder of } \\
\text { DC/AC power }\end{array}$ \\
\hline $\begin{array}{l}\text { Obstructive } \\
\text { hydrocephalus }\end{array}$ & $\begin{array}{l}\text { Increased resistance in CSF path } \\
\text { (due to obstruction) for pulsatile } \\
\text { flow }\end{array}$ & $\begin{array}{l}\text { Increased resistance in CSF } \\
\text { path for AC power }\end{array}$ \\
\hline Ventriculomegaly & $\begin{array}{l}\text { Adaptation by decreased } \\
\text { resistance (due to increased } \\
\text { volume) in CSF path for pulsatile } \\
\text { flow }\end{array}$ & $\begin{array}{l}\text { Adaptation by decreased } \\
\text { resistance in ventricular CSF } \\
\text { path for AC power }\end{array}$ \\
\hline $\begin{array}{l}\text { Pseudotumor } \\
\text { cerebri }\end{array}$ & $\begin{array}{l}\text { Increased resistance in } \\
\text { capillaries due to cerebrovenous } \\
\text { hypertension. CSF pathways are } \\
\text { unobstructed so no windkessel } \\
\text { impairment and therefore no } \\
\text { adaptive ventriculomegaly }\end{array}$ & $\begin{array}{l}\text { Increased resistance to DC } \\
\text { power in capillary path. No } \\
\text { impairment to AC power in CSF } \\
\text { pathways and therefore no } \\
\text { windkessel impairment and no } \\
\text { need for adaptive } \\
\text { ventriculomegaly }\end{array}$ \\
\hline $\begin{array}{l}\text { Low pressure } \\
\text { hydrocephalus }\end{array}$ & $\begin{array}{l}\text { Increased resistance in CSF path } \\
\text { (due to obstruction) for pulsatile } \\
\text { flow combined with increased } \\
\text { intracranial compliance }\end{array}$ & $\begin{array}{l}\text { Increased resistance and } \\
\text { increased compliance in CSF } \\
\text { path for AC power }\end{array}$ \\
\hline
\end{tabular}




\begin{tabular}{|c|c|c|}
\hline $\begin{array}{l}\text { Treatment of low- } \\
\text { pressure } \\
\text { hydrocephalus }\end{array}$ & $\begin{array}{l}\text { Siphoning CSF decreases CSF } \\
\text { path resistance and neck } \\
\text { wrapping decreases intracranial } \\
\text { compliance, restoring windkessel } \\
\text { effectiveness }\end{array}$ & $\begin{array}{l}\text { Siphoning CSF decreases AC } \\
\text { resistance in CSF path and } \\
\text { neck wrapping decreases } \\
\text { intracranial reactance to AC } \\
\text { power, restoring windkessel } \\
\text { effectiveness }\end{array}$ \\
\hline $\begin{array}{l}\text { CSF diversion } \\
\text { (shunting, } \\
\text { ventriculostomies }\end{array}$ & $\begin{array}{l}\text { - CSF diversion provides a } \\
\text { parallel CSF path for } \\
\text { pulsatile flow, restoring } \\
\text { windkessel effectiveness } \\
\text { - CSF diversion provides } \\
\text { path for drainage of } \\
\text { energy from cranium }\end{array}$ & $\begin{array}{l}\text { - CSF diversion provides } \\
\text { a parallel CSF path for } \\
\text { AC power, restoring } \\
\text { windkessel effectiveness } \\
\text { - CSF diversion provides } \\
\text { path for drainage of } \\
\text { energy from cranium }\end{array}$ \\
\hline Cushing's Reflex & $\begin{array}{l}\text { An adaptive reflex to catastrophic } \\
\text { loss of windkessel function. } \\
\text { Hypertension maintains smooth } \\
\text { capillary perfusion while } \\
\text { bradycardia minimizes pulsatile } \\
\text { frequency. Cushing's Reflex is an } \\
\text { accessory windkessel in } \\
\text { extremis. }\end{array}$ & $\begin{array}{l}\text { An adaptive reflex to } \\
\text { catastrophic loss of windkessel } \\
\text { function. Hypertension } \\
\text { maintains DC capillary power } \\
\text { while bradycardia minimizes } \\
\text { capillary AC power. Cushing's } \\
\text { Reflex is an accessory } \\
\text { windkessel in extremis. }\end{array}$ \\
\hline
\end{tabular}

This proposed windkessel model provides novel insights into the dynamics of cerebral blood flow and intracranial pressure. The cerebral windkessel is a frequency-sensitive energy filter, 
and it differs from the traditional Monro-Kellie model of intracranial dynamics in that it is derived from an analysis of energy dynamics of cerebral perfusion, rather than the dynamics of mass balance. The windkessel admits DC power through the capillaries and diverts AC power through the CSF to the veins. The physiological rationale for the windkessel is suppression of motion of capillary walls and maintenance of pulseless capillary perfusion. Capillary pulsatility is suppressed by coupling capillary expansion and relaxation to an equal but opposite-directed compression and expansion of intracranial arteries, veins, and brain parenchyma. Our study suggests that this intracranial circulation current-the cyclic motion of the brain and CSF-is the cornerstone of intracranial windkessel dynamics.

\section{Clinical applications}

The role of windkessel impairment in disorders of intracranial dynamics is virtually unexplored, and this model of the cerebral windkessel provides a new framework for the study and treatment of disorders of cerebral blood flow and intracranial dynamics. Our analysis suggests that maintenance of adequate cerebral perfusion and management of intracranial hypertension in stroke, head injury, brain edema, and hydrocephalus may depend on the optimal ratio of the parameters of heart rate, and intracranial inertance, compliance and vascular and structural resistance.

This ratio is given by a simple equation for windkessel effectiveness: 


$$
W_{\text {eff }}=\frac{L}{R_{C S F} C}
$$

Which expresses the effectiveness of the cerebral windkessel when it is tuned to the heart rate.

Normal windkessel dynamics is frequency (i.e., heart rate) sensitive, which implies that a system of physiological regulation exists that maintains normal windkessel dynamics despite changes in heart rate and other parameters that occur with daily activities.

The insight that normal windkessel dynamics depends on a system of physiological regulation that maintains a specific ratio of heart rate, inertance, compliance and damping in the cranium suggests the need for a revised understanding of disorders of intracranial dynamics such as traumatic brain swelling, hydrocephalus, pseudotumor cerebri, and stroke in light of this system.

A deeper understanding the windkessel and of the intracranial parameters on which it depends may point to novel pharmacological approaches to the treatment of disorders of intracranial dynamics and cerebral blood flow based on an understanding of the physiological regulation of the windkessel.

This understanding of the windkessel also warrants development and potential redesign of devices used for CSF diversion, such as CSF shunts and ventriculostomies. In addition to providing CSF drainage, these devices participate in windkessel dynamics by altering inertance, compliance, and damping in the cranium. 
From the perspective of windkessel dynamics, CSF drainage is also energy drainage, and CSF drainage can be understood as introduction of an artificial accessory windkessel to intracranial dynamics. It is not unreasonable to infer that the notorious limitations in therapeutic effectiveness of these devices may be due, in part, to the way that shunt devices and drainage catheters alter intracranial energy dynamics associated with the windkessel, which has never been explored.

This mathematical windkessel model may also provide a basis for development of machinelearning devices that continuously and automatically assess the cerebral windkessel (using measurement of the transfer function) to provide an early warning of windkessel impairment and incipient brain swelling. This windkessel monitoring could be incorporated into currently available systems for intracranial pressure monitoring and would provide a more effective means of predicting clinical deterioration due to brain swelling and to impairment of cerebral blood flow.

\section{Conclusions}

The cerebral windkessel is the system by which the arterial pulse in the brain is buffered so that blood flow to brain capillaries is smooth. The windkessel is necessary to protect capillaries, and traditional models of intracranial dynamics do not adequately explain how it works. We analyze the windkessel in experimental dogs and describe a new electrical circuit model that produces dynamics that are quantitatively very similar to the cerebral windkessel. This is the first comparison of this new model to actual windkessel dynamics. We show that the windkessel buffering of the arterial pulse in brain capillaries is accomplished by rhythmic motion of the brain and cerebrospinal fluid during the cardiac cycle. Using this simple model of the cerebral windkessel, we apply the concept of energy flow in the brain and show how several important 
disorders of intracranial dynamics can be explained quite easily and naturally as disorders due to impairment of the cerebral windkessel. This new perspective has major implications for research on new treatment strategies for common life-threatening and difficult-to-treat disorders of intracranial dynamics such as brain swelling, hydrocephalus, pseudotumor cerebri, and Cushing's reflex.

\section{Abbreviations}

ABP: Arterial Blood Pressure

AC: Alternating Current

ARX: Autoregressive with exogenous terms

C: Capacitor

CSF: Cerebrospinal fluid

DC: Direct Current

FFT: Fast Fourier Transform

Hz: Hertz

ICP: Intracranial pressure

L: Inductor

MRI: Magnetic Resonance Imaging

PTC: Pseudotumor cerebri

R: Resistor

$\mathrm{R}_{\text {cap }}$ : Series resistor corresponding to the capillary path

$\mathrm{R}_{\mathrm{csF}}$ : Series resistor corresponding to the CSF path

$\mathrm{R}_{\mathrm{wk}}$ : Real component of the windkessel impedance

$W_{\text {eff: }}$ Windkessel effectiveness

$\mathrm{X}_{\mathrm{CSF}}$ : Imaginary component of the windkessel impedance

$Z_{\text {cranium: }}$ : Combined impedance of the capillary and CSF path 
$\omega_{w k}$ : Optimal heart rate

\section{Ethics approval and consent to participate}

For quantitative validation of this model, we use data from a series of dogs (18-26 kg) previously reported [6]. Our protocol was approved by our Institutional Animal care and Use Committee (Wagshul 2007-1504 IACUC), and the animals were treated humanely in accordance with the "Guide for the care and use of laboratory animals" published by the National Institutes of Health.

\section{Consent for publication}

Not applicable.

\section{Availability of data and materials}

The datasets analyzed during the current study are available from the corresponding author on reasonable request.

\section{Competing interests}

The authors declare that they have no competing interests.

\section{Funding}

No funding was received to support this research study and manuscript.

\section{Authors' contributions}

M.E. originated the theory underlying this cerebral windkessel model, developing the physiological ideas and clinical applications of this model, wrote the main manuscript and table text, and wrote the equations in the Background and Discussion. 
L.Y. was responsible for carrying out the data analysis and mathematical applications using ARX modeling, and generated and wrote the equations in the Methods and Results sections.

R.M. contributed to the discussion and refining of concepts for the cerebral windkessel model, to editing the manuscript, to generating figures and tables, and to securing permissions for figures.

S.F. took part in generating graphics and figures for the manuscript.

P.D. was responsible for carrying out the data analysis and mathematical applications using ARX modeling, and generated and wrote the equations in the Methods and Results sections.

All authors reviewed the manuscript.

\section{Acknowledgements}

Not applicable.

\section{References}

1) M. Egnor, L. Zheng, A. Rosiello, F. Gutman, et al. A Model of Intracranial Pulsations. Pediatr Neurosurg. 2001;35:284-298.

2) Egnor M. The cerebral windkessel as a dynamic pulsation absorber. BIO-Complexity 2019(3):doi:10.5048/BIO-C.2019.3

3) N. Westerhof, J.W. Lankhaar, B.E. Westerhof. The arterial Windkessel. Medical \& Biological Engineering \& Computing. 47, 131-141(2009)

4) O. Frank. Die Grundform des arterielen Pulses erste Abhandlung: mathematische Analyse. Z. Biol. 37: 483-526, 1899.

5) N. Stergiopulos, B.E. Westerhof, N. Westerhof. Total arterial inertance as the fourth element of the windkessel model. American Journal of Physiology. Volume 276, Issue 1. January 1999. Pages H81-H88. https://doi.org/10.1152/ajpheart.1999.276.1.H81 
6) M.E. Wagshul, E.J. Kelly, H.J. Yu, B. Garlick, T. Zimmerman, M.R. Egnor. Resonant and notch behavior in intracranial pressure dynamics. J Neurosurg Pediatr 2009;3(5):354-64

7) E.H. Park, S. Dombrowski, M. Luciano, J.R. Madsen, et al. Alterations of pulsation absorber characteristics in experimental hydrocephalus. Journal of Neurosurgery: Pediatrics. 2010;Vol. 6 No. 2: 159-170

8) E.H. Park, P.K. Eide, D. Zurakowski, J.R. Madsen. Impaired pulsation absorber mechanism in idiopathic normal pressure hydrocephalus: laboratory investigation. $J$ Neurosurgery 2012. Dec117(6):1189-96. Doi:10.3171/2012.9.JNS121227.Epub 2012 Oct 12 .

9) Zou R, Park EH, Kelly EM, Egnor M, Wagshul ME, Madsen JR: Intracranial pressure waves: characterization of a pulsation absorber with notch filter properties using systems analysis: laboratory investigation. J Neurosurg Pediatr. 2008, 2: 83-94. 10.3171/PED/2008/2/7/083.

10) Foltz EL (1984) Hydrocephalus and CSF pulsatility: Clinical and laboratory studies. In: Shapiro K, Marmarou A, Portnoy H, eds. Hydrocephalus. Raven (New York) pp 337-362. 19.

11) Sklar FH, Elashvili I (1977) The pressure-volume function of brain elasticity. Physiological considerations and clinical applications. J Neurosurg 47(5):670-679. doi:10.3171/jns.1977.47.5.0670

12) Wang JJ, O'Brien AB, Schrive NG, Parker KH, et al. Time domain representation of ventricular-arterial coupling as a windkessel and wave system. Am J Physiol Heart Circ Physiol 2003;284:H1358-1368

13) M. Ursino, M. Giulioni, and C. A. Lodi. Relationships among cerebral perfusion pressure, autoregulation, and transcranial Doppler waveform: a modeling study. Journal of neurosurgery, 89(2):255-266, August 1998. 
14) Mauro Ursino and Carlo A. Lodi. A simple mathematical model of the interaction between intracranial pressure and cerebral hemodynamics. Journal of Applied Physiology, 82(4):1256-1269, April 1997

15) Ying Zheng, John Mayhew. A time-invariant visco-elastic windkessel model relating blood flow and blood volume. Volume 47, Issue 4, 1 October 2009, Pages 1371-1380

16) Oppenheim, Alan (2010). Discrete Time Signal Processing Third Edition. Upper Saddle River, NJ: Pearson Higher Education, Inc. p. 504.]

17) Greitz D. Cerebrospinal fluid circulation and associated intracranial dynamics. A radiologic investigation using MR imaging and radionuclide cisternography. Acta Radiol Suppl;1993: 386:1-23

18) Edvinsson L, Hogestatt ED, Uddman R, Auer LM: Cerebral veins: fluorescence histochemistry, electron microscopy, and in vitro reactivity. J Cereb Blood Flow Metab 1983;3:226-230

19) Edvinsson L, McCulloch J, Uddman R: Feline cerebral veins and arteries: comparison of autonomic innervation and vasomotor responses. J Physiol. 325:161-173, 1982

20) Dr F. Gjerris MD, PhD P. Soelberg Sørensen MD S. Vorstrup MD O. B. Paulson MD, $\mathrm{PhD}$ Intracranial pressure, conductance to cerebrospinal fluid outflow, and cerebral blood flow in patients with benign intracranial hypertension (pseudotumor cerebri). Annals of Neurology. Volume 17, Issue 2. February 1985. Pages 158-162

21) Kalani, M. Yashar S., Jay D. Turner, and Peter Nakaji. "Treatment of refractory lowpressure hydrocephalus with an active pumping negative-pressure shunt system." Journal of Clinical Neuroscience 20.3 (2013): 462-466.

22) Harald Fodstad, M.D., Ph.D., Patrick J. Kelly, M.D., Michael Buchfelder, M.D. History of the Cushing Reflex. Neurosurgery, Volume 59, Issue 5, November 2006, Pages 1132 1137 


\section{Figures}

\section{Figure 1}

Graphs showing the transfer function of the windkessel in an experimental dog (from Wagshul et. al. [6] with permission)

(A) The amplitude transfer function, which shows the high impedance stop band at the heart rate. This represents the suppression of the arterial pulse in the capillaries of the brain parenchyma. (B) The phase transfer function, which shows a phase transition near the cardiac fundamental, which is characteristic of a pulsation absorber tuned to high impedance resonance at the heart rate. The open circles mark the fundamental (heart rate) harmonic, and the second and third harmonics. The electrical analogue of the cerebral windkessel is a tank circuit with capacitance and inductance in parallel (see text).

Figure 2
A) The experimentally measured ICP lead with respect to the arterial pulse in a dog from Wagshul et al.[6]
B) Graphical evidence that the phase lead of the ICP with respect to the ABP is real, and not merely a five- sixth cycle lag from the previous ABP pulse. 


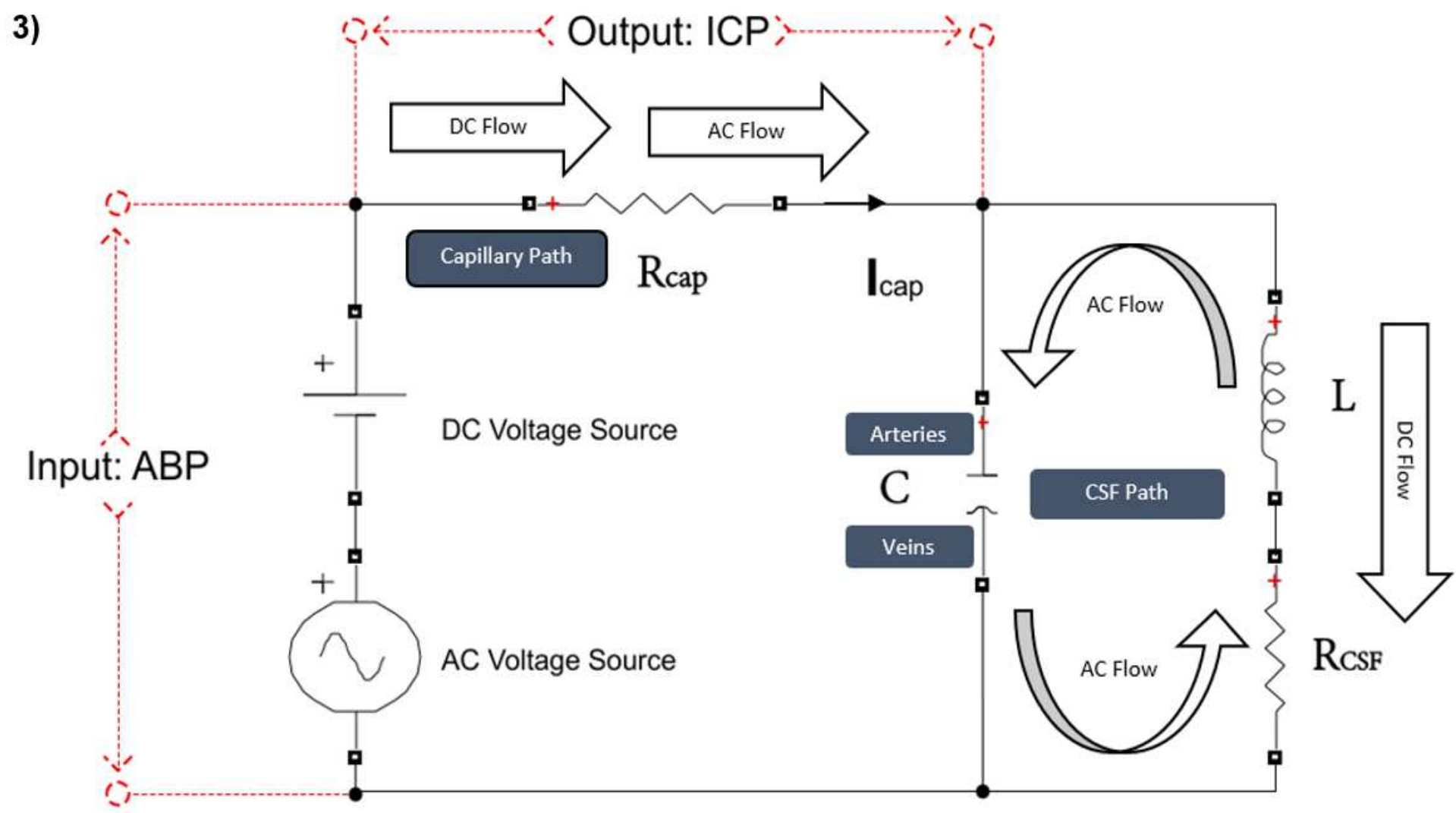

Figure 3

Windkessel circuit model. The model clarifies the dynamics of the cerebral windkessel. Suppression of the AC current from the capillary path is accomplished by cyclic loading and unloading of the 'arterial' and 'venous' capacitor plates, which continuously opposes and blocks the cyclic current from the AC arterial source. The tank circuit has a DC and AC source (corresponding in the cranium to MAP and pulse pressure, respectively), a series resistor (corresponding to the capillary path), and a parallel RLC circuit (corresponding to the CSF, interstitial fluid, brain parenchyma, and intracranial arteries and veins, which for brevity we call the CSF path). Voltage represents pressure, current represents flow, and charge represents volume. Voltage across the source is a proxy for $A B P$, and voltage across the capillary resistor is a proxy for ICP. Capacitance represents the compliance of the extracapillary (parenchymal and CSF) path and corresponds primarily to the compliance of the intracranial veins. Inductance represents intracranial inertance, which corresponds to the inertance of the extracapillary cranial contents. represents structural damping of the vascular walls and brain parenchyma and resistance to compression/expansion of the cerebral arteries and veins. is effectively a venous pump. corresponds to intracapillary resistance to longitudinal blood flow, and is the net resistance in the extracapillary space to radial expansion and relaxation of capillary walls. Note that does not represent the resistance to CSF absorption. The DC power that drives CSF formation and absorption is several orders of magnitude smaller than $\mathrm{AC}$ power ${ }^{1}$ and is represented in this model as a miniscule component of $\mathrm{DC}$ power. 
Circulation flow of the cerebral windkessel and suppression of capillary AC power Suppression of the arterial pulse in the capillaries is accomplished in the cranium by a cyclic exchange of volume between the intracranial arteries and veins, linked by the CSF. This is analogous to the circulation current in a tank circuit, which represents the same dynamics. At every moment through the cardiac cycle, the flow of volume in the cerebral windkessel (the extracapillary cranial contents) is equal to and opposite the arterial pulse entering the capillary bed, which cancels the pulse in the arterial inflow to the capillaries and diverts it through the CSF to the intracranial veins. This cyclic exchange of volume between the intracranial arteries and veins can be simulated in the tank circuit as the loading and unloading of charge on the 'arterial' and 'venous' plates of the capacitor in the tank (Fig 3), and it renders capillary blood flow (nearly) pulseless.
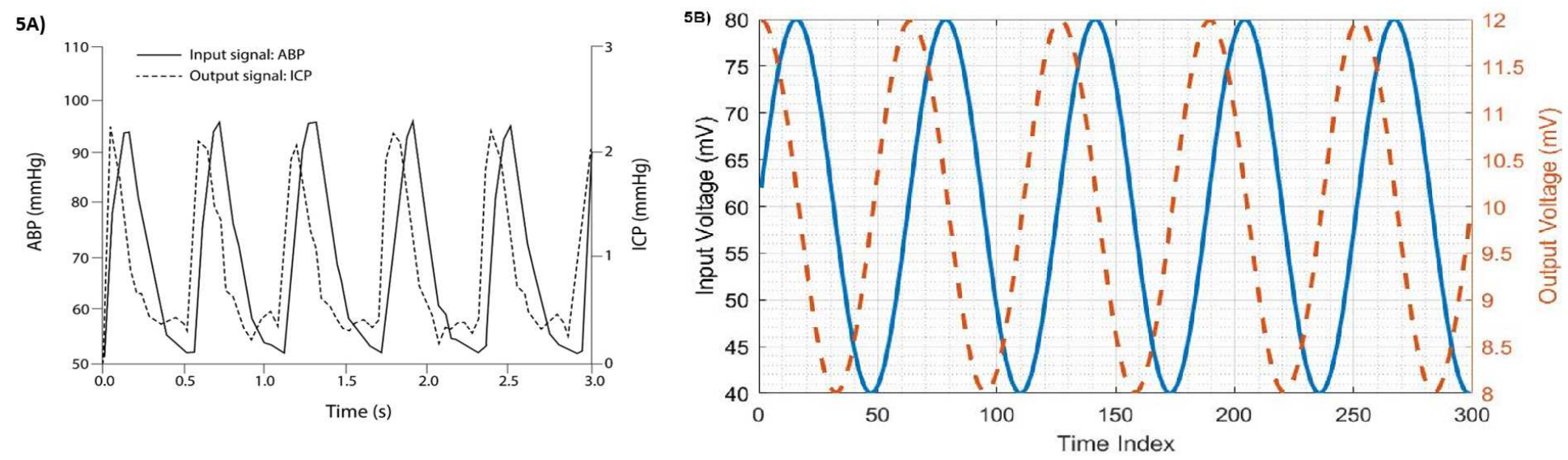

Figure 5

$A$ and $B$ : The phase lead of ICP with respect to ABP in the cerebral windkessel. Fig $5 A$ shows the experimentally measured ICP lead with respect to the arterial pulse in a dog from Wagshul et al [6]. Fig 5B shows the lead of the voltage across the main resistor (which is the ICP proxy) in the tank circuit model and the voltage across the source (which is the ABP proxy). In Fig 5B, the solid line is the ABP and the dotted line is the ICP, as in Fig $5 \mathrm{~A}$.

Figure 6

The comparison of the transfer functions of the windkessel model and the dog data. Given the dog data, the transfer function from the output ICP to input ABP can be obtained directly by computing the FFT. According to the model, the transfer functions of the ARX and circuit models should capture the salient features of the transfer function from the dog data. The transfer function of the circuit should be approximately equivalent to the ARX model near the heart rate frequency. In this figure, we show results of four examples with normal mean ICP. 


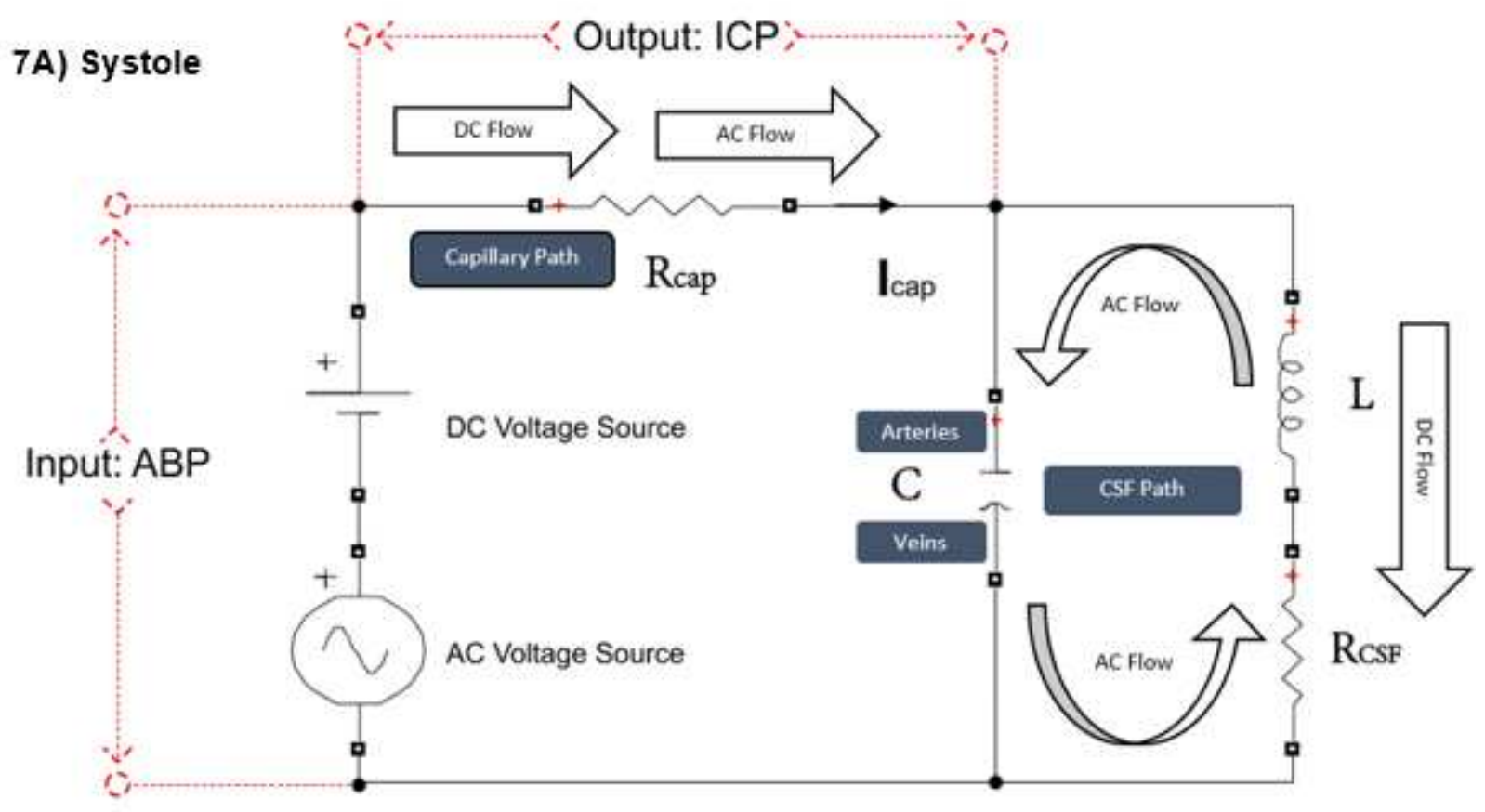

7B) Diastole

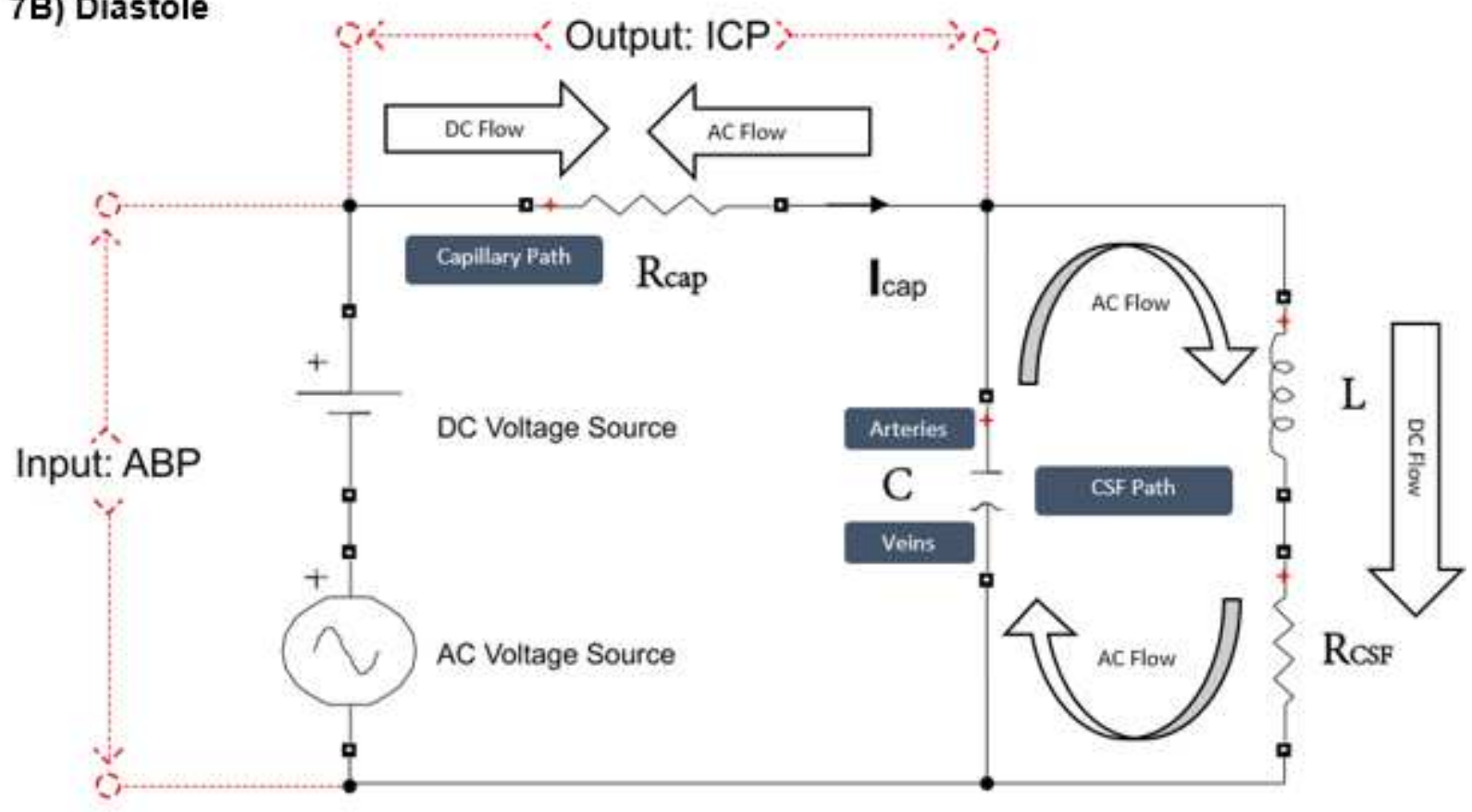

Figure 7

Windkessel circuit model in systole and diastole

The tank circuit has a DC and AC source (corresponding in the cranium to MAP and pulse pressure, respectively), a series resistor $R_{\text {cap }}$ (corresponding to the capillary path), and a parallel RLC circuit (corresponding to the CSF, interstitial fluid, brain parenchyma, and intracranial arteries and veins, which for brevity we call the CSF path). Voltage represents pressure, current represents flow, and charge represents volume. Voltage across the source is a proxy for $A B P$, and voltage across the capillary resistor is a proxy for ICP. Capacitance $C$ represents the compliance of the extracapillary (parenchymal and CSF) 
path and corresponds primarily to the compliance of the intracranial veins. Inductance $L$ represents intracranial inertance, which corresponds to the inertance of the extracapillary cranial contents. $R_{\mathrm{CFS}}$ represents structural damping of the vascular walls and brain parenchyma and resistance to compression/expansion of the cerebral arteries and veins. $R_{C F S}$ is effectively a venous pump. $\mathbf{R}_{\text {cap }}$ corresponds to intracapillary resistance to longitudina/ blood flow, $\mathbf{R}_{\mathrm{CFS}}$ and is the net resistance in the extracapillary space to radial expansion and relaxation of capillary walls. Note that RCFS does not represent the resistance to CSF absorption. The DC power that drives CSF formation and absorption is several orders of magnitude smaller than AC power [1] and is represented in this model as a miniscule component of DC power.

The model clarifies the dynamics of the cerebral windkessel. Suppression of the AC current from the capillary path is accomplished by cyclic loading and unloading of the 'arterial' and 'venous' capacitor plates, which continuously opposes and blocks the cyclic current from the AC arterial source. This circulation current is the motion of the brain and CSF in the cardiac cycle-in systole the brain expands and moves caudally, and in diastole it relaxes and rebounds cranially [17]. This accomplishes the cerebral windkessel.

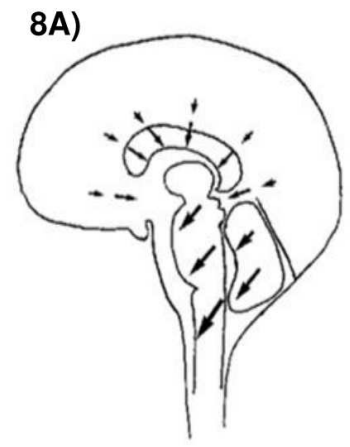

a

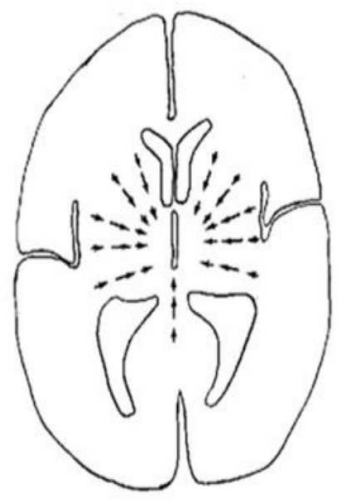

$c$

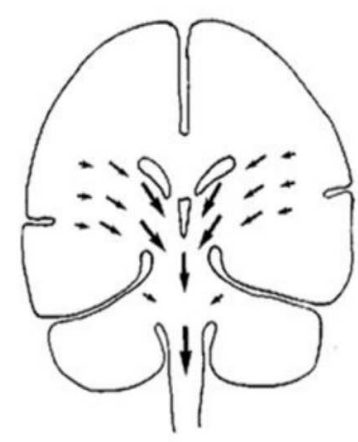

b

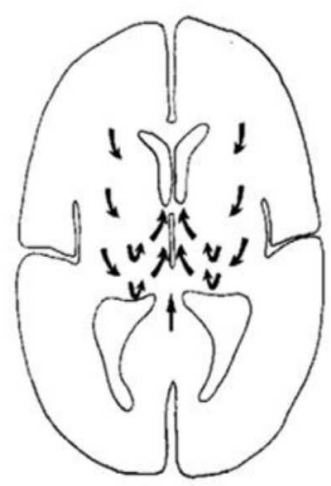

d
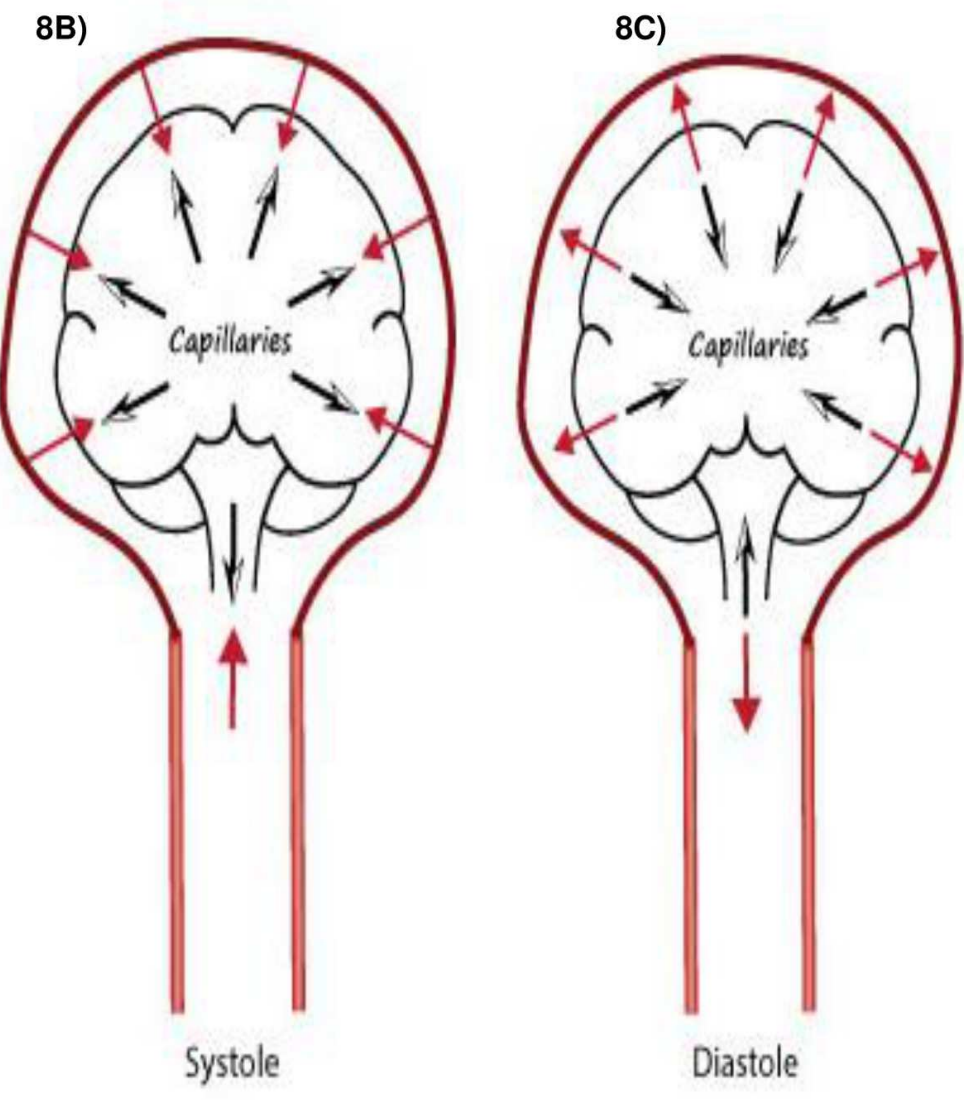

Figure 8 
Implementation of the cerebral windkessel by cyclic brain, CSF, and vascular motion during the cardiac cycle.

8A) Using flow MRI imaging, Greitz ${ }^{17}$ has shown that during systole the brain undergoes two motions: centrifugal expansion and caudal displacement toward the skull base. During diastole, the brain relaxes centripetally, and rebounds cranially. This motion is represented in 4 panels, a-d (from Greitz et al. [17] with permission).

8B) Motion of the brain in systole. In systole, the arterial pulse enters the cranium via the circle of Willis at the skull base and enters the brain via perforating arteries that enter the parenchyma at right angles to the cortical surface. Note that the motion of the brain during systole continuously opposes the systolic pulsatile bolus of arterial blood. The systolic arterial bolus is opposed by simultaneous downward displacement of the brain against the arterial expansion in the circle of Willis, and centrifugal expansion of the brain opposes the arterial bolus entering via the perforating arteries entering the cortex.

The systolic arterial pulse is depicted by red solid arrows, and the centrifugal and caudal brain motion is depicted by partially open arrows. In systole, brain expansion and caudal brain displacement deflects a portion of the systolic arterial pulse away from the capillary circulation and diverts the pulsatile energy through the CSF spaces to the veins.

8C) Motion of the brain in diastole. The diastolic arterial pulse is depicted by red solid arrows, and the centripetal and cranial brain motion is depicted by partially open arrows. In diastole, centripetal and caudal brain relaxation opposes diastolic relaxation of the arteries, which augments the capillary circulation and returns a portion of the systolic kinetic energy to the diastolic capillary flow. 